Article

\title{
Lie Symmetry Analysis of the Hopf Functional-Differential Equation
}

\section{Daniel D. Janocha *, Marta Wacławczyk ${ }^{\dagger}$ and Martin Oberlack ${ }^{\dagger}$}

Chair of Fluid Dynamics, Department of Mechanical Engineering, TU Darmstadt, Otto-Berndt-Str. 2, Darmstadt 64287, Germany; E-Mails: martaw@fdy.tu-darmstadt.de (M.W.);

oberlack@fdy.tu-darmstadt.de (M.O.)

${ }^{\dagger}$ These authors contributed equally to this work.

* Author to whom correspondence should be addressed; E-Mail: danieljanocha@gmx.de

Academic Editor: Sergei D. Odintsov

Received: 17 July 2015 / Accepted: 20 August 2015 / Published: 27 August 2015

\begin{abstract}
In this paper, we extend the classical Lie symmetry analysis from partial differential equations to integro-differential equations with functional derivatives. We continue the work of Oberlack and Wacławczyk (2006, Arch. Mech. 58, 597), (2013, J. Math. Phys. 54, 072901), where the extended Lie symmetry analysis is performed in the Fourier space. Here, we introduce a method to perform the extended Lie symmetry analysis in the physical space where we have to deal with the transformation of the integration variable in the appearing integral terms. The method is based on the transformation of the product $y(x) \mathrm{d} x$ appearing in the integral terms and applied to the functional formulation of the viscous Burgers equation. The extended Lie symmetry analysis furnishes all known symmetries of the viscous Burgers equation and is able to provide new symmetries associated with the Hopf formulation of the viscous Burgers equation. Hence, it can be employed as an important tool for applications in continuum mechanics.
\end{abstract}

Keywords: Lie symmetries; Hopf equation; Burgers equation; functional differential equations; turbulence 


\section{Introduction}

\subsection{Three Complete Descriptions of Turbulence}

Turbulence research knows three complete descriptions of turbulence. In each of these descriptions, the aim is to calculate the statistics of a turbulent flow by determining the so-called multi-point velocity correlations or simply multi-point correlations:

$$
\left\langle U_{i_{1}}^{t}\left(\boldsymbol{x}_{1}\right) \ldots U_{i_{n}}^{t}\left(\boldsymbol{x}_{n}\right)\right\rangle, \quad i_{1}, \ldots, i_{n}=1,2,3
$$

In this term, $\boldsymbol{U}^{t}$ is a random vector describing the velocity field in time $t$, and $\boldsymbol{x}_{1}, \ldots, \boldsymbol{x}_{n}$ are the positions of different fluid particles in the space occupied by the fluid. $\boldsymbol{U}^{t}$ is given by:

$$
\boldsymbol{U}^{t}\left(\boldsymbol{x}_{i}\right)=\left(U_{1}^{t}\left(\boldsymbol{x}_{i}\right), U_{2}^{t}\left(\boldsymbol{x}_{i}\right), U_{3}^{t}\left(\boldsymbol{x}_{i}\right)\right)=\left(U_{1}\left(\boldsymbol{x}_{i}, t\right), U_{2}\left(\boldsymbol{x}_{i}, t\right), U_{3}\left(\boldsymbol{x}_{i}, t\right)\right)
$$

In the language of stochastics, the multi-point correlations are the covariances of the velocity components. We briefly introduce three complete descriptions of turbulence research.

- In the multi-point correlation approach, an infinite dimensional chain of linear, but non-local differential equations has to be solved. On the $n$-th level, the unknown $(n+1)$-point correlation is present. Solving the infinitely many equations directly provides all multi-point correlations. In [1], the Lie symmetries of the infinite set of multi-point correlation equations are investigated.

- In the Lundgren-Monin-Novikov approach [2], it is assumed that the velocity field $\boldsymbol{U}^{t}$ admits probability density functions (PDFs):

$$
f_{n}^{t}:=f^{t}\left(\boldsymbol{v}_{1}, \ldots, \boldsymbol{v}_{n} ; \boldsymbol{x}_{1}, \ldots, \boldsymbol{x}_{n}\right)
$$

given in terms of the Dirac delta distribution and describing the correlation of the velocity components at multiple points in space. To be more precise, $f^{t}\left(\boldsymbol{v}_{1} ; \boldsymbol{x}_{1}\right) \mathrm{d} \boldsymbol{v}_{1}$ expresses the probability that the velocity vector $\boldsymbol{U}^{t}\left(\boldsymbol{x}_{1}\right)=\boldsymbol{U}\left(\boldsymbol{x}_{1}, t\right)$ is contained within the infinitesimal interval $\left[\boldsymbol{v}_{1}, \boldsymbol{v}_{1}+\mathrm{d} \boldsymbol{v}_{1}\right]$. The Lundgren-Monin-Novikov hierarchy is an infinite dimensional chain of non-local differential equations for the PDFs, where on the $n$-th level, the unknown $(n+1)$-point PDF $f_{n+1}^{t}=f^{t}\left(\boldsymbol{v}_{1}, \ldots, \boldsymbol{v}_{(n+1)} ; \boldsymbol{x}_{1}, \ldots, \boldsymbol{x}_{(n+1)}\right)$ is present. Solving the infinitely many equations provides all PDFs. The multi-point correlations can be calculated by integrating the PDFs via:

$$
\left\langle U_{i_{1}}^{t}\left(\boldsymbol{x}_{1}\right) \ldots U_{i_{n}}^{t}\left(\boldsymbol{x}_{n}\right)\right\rangle=\int_{\mathbb{R}^{3}} \ldots \int_{\mathbb{R}^{3}} v_{i_{1}} \ldots v_{i_{n}} f^{t}\left(\boldsymbol{v}_{1}, \ldots, \boldsymbol{v}_{n} ; \boldsymbol{x}_{1}, \ldots, \boldsymbol{x}_{n}\right) \mathrm{d} \boldsymbol{v}_{1} \ldots \mathrm{d} \boldsymbol{v}_{n}
$$

In [3], the Lie symmetries of the Lundgren-Monin-Novikov hierarchy are investigated.

- In the Hopf approach, the characteristic functions $\phi$ of the PDFs $f^{t}$ for $n \rightarrow \infty$ are investigated, $c f$. [4]. The $n$-point characteristic function is defined as:

$$
\phi\left(\boldsymbol{y}_{1}, \ldots, \boldsymbol{y}_{n}, t\right):=\left\langle e^{i\left(\mathcal{U}^{t}, \boldsymbol{y}\right)}\right\rangle=\int_{\mathbb{R}^{3}} \ldots \int_{\mathbb{R}^{3}} e^{i(\boldsymbol{v}, \boldsymbol{y})} f^{t}\left(\boldsymbol{v}_{1}, \ldots, \boldsymbol{v}_{n} ; \boldsymbol{x}_{1}, \ldots, \boldsymbol{x}_{n}\right) \mathrm{d} \boldsymbol{v}_{1} \ldots \mathrm{d} \boldsymbol{v}_{n}
$$

with $\mathcal{U}^{t}:=\left(\boldsymbol{U}^{t}\left(\boldsymbol{x}_{1}\right), \ldots, \boldsymbol{U}^{t}\left(\boldsymbol{x}_{n}\right)\right) .(\cdot, \cdot)$ is defined as the Euclidean scalar product

$$
(\boldsymbol{v}, \boldsymbol{y}):=\sum_{k=1}^{n} \sum_{i=1}^{3} v_{k_{i}} y_{k_{i}}
$$

for $\boldsymbol{v}=\left(\boldsymbol{v}_{1}, \ldots, \boldsymbol{v}_{n}\right), \boldsymbol{y}=\left(\boldsymbol{y}_{1}, \ldots, \boldsymbol{y}_{n}\right)$ with $\boldsymbol{v}_{k}, \boldsymbol{y}_{k} \in \mathbb{R}^{3}, k=1, \ldots, n$. 
In hydromechanics, we assume that the mean free path is negligible; hence, we take the continuum limit $n \rightarrow \infty$. Thus, instead of the $n$ velocity vectors $\boldsymbol{v}_{1}, \ldots, \boldsymbol{v}_{n}$, we consider a continuous set $[\boldsymbol{v}(\boldsymbol{x})]$, such that the velocity $\boldsymbol{v}$ is a continuous function depending on the spatial variable $\boldsymbol{x}$. In the continuum limit, the probability density function $f^{t}$ becomes a probability density functional, and the function $\phi$ becomes the functional:

$$
\phi([\boldsymbol{y}(\boldsymbol{x})], t)=\left\langle e^{i\left(\boldsymbol{U}^{t}, \boldsymbol{y}\right)}\right\rangle=\int e^{i(\boldsymbol{v}, \boldsymbol{y})} f^{t}([\boldsymbol{v}(\boldsymbol{x})]) \mathrm{d}[\boldsymbol{v}(\boldsymbol{x})]
$$

In this paper, we pursue the Hopf approach defined in Equation (1). A relation between the Hopf and Lundgren approach is discussed in [5], where also equations in a Lagrangian multi-particle framework are investigated.

\subsection{Hopf Functional and Multi-Point Correlations}

In Equation (1), it is not directly apparent what is meant by $(\cdot, \cdot)$ and how the integration domain should be chosen. In order to define $\phi=\phi([\boldsymbol{y}(\boldsymbol{x})], t)$ properly, we introduce the $L^{2}$ space.

Definition 1 ( $L^{2}$ space). For $G \subseteq \mathbb{R}^{3}$, define

$$
L^{2}\left(G, \mathbb{R}^{3}\right):=\left\{\boldsymbol{v}: G \longrightarrow \mathbb{R}^{3} \mid \int_{G} \boldsymbol{v}(\boldsymbol{x}) \cdot \boldsymbol{v}(\boldsymbol{x}) \mathrm{d} \boldsymbol{x}=\int_{G} \sum_{i=1}^{3} v_{i}(\boldsymbol{x}) v_{i}(\boldsymbol{x}) \mathrm{d} \boldsymbol{x}<\infty\right\}
$$

and equip $L^{2}\left(G, \mathbb{R}^{3}\right)$ with $(\cdot, \cdot)$ defined by:

$$
(\boldsymbol{v}, \boldsymbol{y}):=\int_{G} \boldsymbol{v}(\boldsymbol{x}) \cdot \boldsymbol{y}(\boldsymbol{x}) \mathrm{d} \boldsymbol{x}=\int_{G} \sum_{i=1}^{3} v_{i}(\boldsymbol{x}) y_{i}(\boldsymbol{x}) \mathrm{d} \boldsymbol{x}
$$

for $\boldsymbol{v}, \boldsymbol{y} \in L^{2}\left(G, \mathbb{R}^{3}\right)$.

If we equip $L^{2}\left(G, \mathbb{R}^{3}\right)$ with $(\cdot, \cdot)$ defined by Equation (2), it is a Hilbert space; $c f$. [6].

Definition 2 (Hopf functional, Hopf functional differential equation). Let $\boldsymbol{y} \in L^{2}\left(G, \mathbb{R}^{3}\right)$, and let $(\cdot, \cdot)$ be the $L^{2}$ scalar product defined by Equation (2). The characteristic functional or Hopf functional is defined by:

$$
\phi([\boldsymbol{y}(\boldsymbol{x})], t):=\left\langle e^{i\left(\boldsymbol{U}^{t}, \boldsymbol{y}\right)}\right\rangle=\int_{L^{2}\left(G, \mathbb{R}^{3}\right)} e^{i(\boldsymbol{v}, \boldsymbol{y})} f^{t}([\boldsymbol{v}(\boldsymbol{x})]) \mathrm{d}[\boldsymbol{v}(\boldsymbol{x})]
$$

Instead of dealing with an infinite dimensional chain of differential equations, the Hopf approach works with one scalar functional differential equation (FDE).

An FDE is a generalization of a partial differential equation (PDE). A PDE becomes an FDE if the number of independent variables tends to infinity. Precisely:

Definition 3 (Functional, functional derivative, functional differential equation).

- Let $\mathcal{F}$ be a function space. A functional is a mapping:

$$
\phi: \mathcal{F} \longrightarrow \mathbb{R}
$$


- Let $\phi=\phi([\boldsymbol{y}(\boldsymbol{x})])$ be a functional. We define the functional derivative of $\phi$ as the limit:

$$
\frac{\partial \phi([\boldsymbol{y}(\boldsymbol{x})])}{\partial y_{\alpha}(\boldsymbol{z}) \mathrm{d} \boldsymbol{z}}:=\lim _{h \rightarrow 0} \frac{\phi\left(\left[\boldsymbol{y}(\boldsymbol{x})+h \boldsymbol{e}_{\alpha} \delta(\boldsymbol{x}-\boldsymbol{z})\right]\right)-\phi([\boldsymbol{y}(\boldsymbol{x})])}{h}, \quad \alpha=1,2,3
$$

Here, $\boldsymbol{e}_{\alpha}$ denotes the $\alpha$-th unit vector and $\delta$ denotes the Dirac delta distribution. (In fact, this is a corollary of the definition of the Gâteaux derivative; cf. [7]. As the presented formula is more suitable for calculations, we use it as definition.) We denote the functional derivative by:

$$
\phi_{, y_{\alpha}(\boldsymbol{z})}=\frac{\partial \phi}{\partial y_{\alpha}(\boldsymbol{z}) \mathrm{d} \boldsymbol{z}}=\frac{\delta \phi}{\delta y_{\alpha}(\boldsymbol{z})}
$$

- Let $\phi=\phi\left(\left[\boldsymbol{y}\left(\boldsymbol{x}^{\prime}\right)\right], \boldsymbol{x}, t\right)$ be a functional. A functional differential equation (FDE) of order $q$ is an equation $F=0$ where $F$ is a functional relating $\phi$ and all its derivatives up to order $q$, which can include partial derivatives with respect to $t$, partial derivatives with respect to $x$ and functional derivatives with respect to each $y_{\alpha}\left(\boldsymbol{x}^{\prime}\right), \alpha=1,2,3$ :

$$
F\left(\left[\boldsymbol{y}\left(\boldsymbol{x}^{\prime}\right)\right], \boldsymbol{x}, t, \boldsymbol{\phi} \underset{1}{\phi}, \underset{2}{\phi}, \ldots, \underset{q}{\phi}\right)=0
$$

In an FDE, the finite set $\left(\boldsymbol{y}_{1}, \ldots, \boldsymbol{y}_{n}\right) \in\left(\mathbb{R}^{3}\right)^{n}$ is replaced by an infinite set $\left[\boldsymbol{y}\left(\boldsymbol{x}^{\prime}\right)\right] \in \mathcal{F}$ with a continuous parameter $\boldsymbol{x}^{\prime}$ representing the continuum analogon of the discrete counting parameter $k=1, \ldots, n$. Hence, the dependent variables are functionals, as they depend on functions.

It is important to note that we may obtain all multi-point correlations by differentiating $\phi$ and evaluating the derivatives at $\boldsymbol{y}=\mathbf{0}$ via:

$$
\left\langle U_{\alpha_{1}}^{t}\left(\boldsymbol{x}_{1}\right) \ldots U_{\alpha_{n}}^{t}\left(\boldsymbol{x}_{n}\right)\right\rangle=\left.\frac{1}{i^{n}} \phi_{, y_{\alpha_{n}}\left(\boldsymbol{x}_{n}\right) \ldots y_{\alpha_{1}}\left(\boldsymbol{x}_{1}\right)}\right|_{\boldsymbol{y}=\mathbf{0}}
$$

Consequently, the Hopf functional $\phi$ provides the full statistical description of velocity field $\boldsymbol{U}^{t}$, since all multi-point correlations can be expressed in terms of $\phi$ and its functional derivatives.

In order to solve FDEs containing the Hopf functional $\phi$, so-called Hopf FDEs, we use an extension of the classical Lie symmetry analysis. The extension is done in [8], where it is shown how the Lie symmetry analysis is performed with FDEs. The extended Lie symmetry analysis is applied to the Hopf formulation of the viscous and inviscid Burgers equation in [9].

In order to circumvent the partial derivatives $\partial / \partial x$ appearing both in the viscous and in the inviscid Hopf-Burgers FDE, in [8,9], the extended Lie symmetry analysis is performed in Fourier space by considering the Fourier transform of the Hopf-Burgers FDE. In the Fourier space, derivatives with respect to $x$ become multipliers with $i k$. Hence, in [8,9], $k$ was not transformed separately, but was treated as a continuous index. In [9], we also derived invariant solutions for the Hopf equations; however, due to the restriction of the method (no transformation of $k$ ), the solutions contained unknown functions of $k$ governed by an infinite chain of equations. We note here that the Hopf-Burgers functional was also considered in [10,11], where, using a numerical integration, scaling in $k$ and the decay of energy was calculated. As far as symmetry methods are concerned, interesting aspects of energy scaling for turbulence were discovered in [12] based on self-similar solutions of the Leith model.

In this paper, the extended Lie symmetry analysis is performed on the viscous Hopf-Burgers FDE in the physical space. Hence, we have to deal with partial derivatives with respect to $x$. There are several approaches for how to do that. The approaches are introduced in Section 3.1. 


\subsubsection{Viscous Hopf-Burgers Functional Integro-Differential Equation}

Our primary long-term goal is to investigate the Hopf functional of turbulent velocity, i.e., the Hopf-Navier-Stokes FDE. For the sake of convenience, we presently restrict ourselves to the one-dimensional case, i.e., replace $\mathbb{R}^{3}$ by $\mathbb{R}$. Instead of the incompressible Navier-Stokes equations, we use the viscous Burgers equation to derive the so-called viscous Hopf-Burgers FDE. We assume that $y, U^{t} \in L^{2}(G, \mathbb{R})$ with

$$
y=y(x), \quad U^{t}=U^{t}(x)=U(x, t),
$$

and that $U^{t}$ fulfills the viscous Burgers equation:

$$
\frac{\partial U^{t}}{\partial t}+U^{t} \frac{\partial U^{t}}{\partial x}=v \frac{\partial^{2} U^{t}}{\partial x^{2}}
$$

which can equivalently by written as

$$
\frac{\partial U^{t}}{\partial t}+\frac{1}{2} \frac{\partial\left(U^{t}\right)^{2}}{\partial x}=v \frac{\partial^{2} U^{t}}{\partial x^{2}}
$$

By simple rescaling, we may eliminate the factor $1 / 2$. We call the following equation the viscous Burgers equation:

$$
\frac{\partial U^{t}}{\partial t}+\frac{\partial\left(U^{t}\right)^{2}}{\partial x}=v \frac{\partial^{2} U^{t}}{\partial x^{2}}
$$

We differentiate the Hopf functional (3) with respect to $t$ to get:

$$
\phi_{, t}([y(x)], t)=\frac{\partial}{\partial t}\left\langle e^{i\left(U^{t}, y\right)}\right\rangle=\left\langle i\left(\frac{\partial U^{t}}{\partial t}, y\right) e^{i\left(U^{t}, y\right)}\right\rangle=\left\langle i\left(-\frac{\partial\left(U^{t}\right)^{2}}{\partial x}+v \frac{\partial^{2} U^{t}}{\partial x^{2}}\right) e^{i\left(U^{t}, y\right)}\right\rangle
$$

where Equation (6) was inserted in order to eliminate $\partial U^{t} / \partial t$. Using the definition of the characteristic functional (1), the following relations can be calculated:

$$
\begin{aligned}
-\left\langle\frac{\partial\left(U^{t}\right)^{2}}{\partial x} e^{i\left(U^{t}, y\right)}\right\rangle & =\frac{\partial}{\partial x} \frac{\delta^{2} \phi}{\delta y(x) \delta y(x)}=\phi_{, x y(x) y(x)} \\
i \nu\left\langle\frac{\partial^{2} U^{t}}{\partial x^{2}} e^{i\left(U^{t}, y\right)}\right\rangle & =v \frac{\partial}{\partial x^{2}} \frac{\delta \phi}{\delta y(x)}=v \phi_{, x x y(x)}
\end{aligned}
$$

After introducing the above formulas into Equation (7), we finally obtain the viscous Hopf-Burgers FDE:

$$
\phi_{, t}([y(x)], t)=\int_{G} y(x)\left(i \phi_{, x y(x) y(x)}+\boldsymbol{v} \phi_{, x x y(x)}\right) \mathrm{d} x
$$

with $y \in L^{2}(G, \mathbb{R})$. Since $\phi$ is part of the integrand, the viscous Hopf-Burgers FDE is an integro-differential equation.

In the following, we want to perform the extended Lie symmetry analysis on the viscous Hopf-Burgers FDE. Before doing that, we review the extended Lie symmetry analysis developed in $[8,9]$ and advance it for our purposes.

This paper is structured as follows: In the second section, we present an extension of the classical Lie symmetry analysis based on the two papers [8,9], which allows us to analyze functional equations 
in physical space. The third section presents the main part of the paper: we apply the extended Lie symmetry analysis to the viscous Hopf-Burgers functional integro-differential equation. First, we present three different approaches to perform the extended Lie symmetry analysis on the viscous Hopf-Burgers FDE. Subsequently, we solve the system of equations for the infinitesimals. Then, we discuss symmetry breaking restrictions and indicate physically-relevant symmetries. At the end of the third section, we compare the symmetries of the viscous Hopf-Burgers FDE with the symmetries of the viscous Burgers equation and calculate the associated global transformations. Finally, a conclusion and perspectives are given in the fourth section.

\section{Extension of the Lie Symmetry Analysis towards Functional Integro-Differential Equations}

\subsection{One-Parameter Lie Point Transformations}

To start with, we introduce some basic notions; $c f$. [8]. As in this paper we consider the viscous Hopf-Burgers FDE being of third order, we set $q=3$ in Equation (4). Furthermore, as we use the viscous Burgers equation as the underlying equation for fluid motion instead of the incompressible Navier-Stokes equations, we replace $\mathbb{R}^{3}$ by $\mathbb{R}$. The whole theory can easily be extended to higher dimensions and to differential equations of arbitrary order $q$.

In Lie symmetry analysis, we only consider continuous symmetry transformations depending on a continuous parameter $\epsilon \in S$ where $S$ is a Lie group. We restrict ourselves to so-called one-parameter Lie point transformations; $c f$. [13]:

Definition 4 (One-parameter Lie point transformations of PDEs). Consider the PDE:

$$
F\left(y_{1}, \ldots, y_{n}, x_{1}, \ldots, x_{n}, t, \underset{1}{\phi} \underset{2}{\phi}, \ldots, \underset{3}{\phi}\right)=0
$$

and let:

$$
\begin{array}{rlrl}
\overline{y_{i}} & =\overline{y_{i}}\left(y_{1}, \ldots, y_{n}, x_{1}, \ldots, x_{n}, t, \phi, \epsilon\right), & & i=1, \ldots, n, \\
\overline{x_{i}} & =\overline{x_{i}}\left(y_{1}, \ldots, y_{n}, x_{1}, \ldots, x_{n}, t, \phi, \epsilon\right), & & i=1, \ldots, n, \\
\bar{t} & =\bar{t}\left(y_{1}, \ldots, y_{n}, x_{1}, \ldots, x_{n}, t, \phi, \epsilon\right), & \\
\bar{\phi} & =\bar{\phi}\left(y_{1}, \ldots, y_{n}, x_{1}, \ldots, x_{n}, t, \phi, \epsilon\right) &
\end{array}
$$

be the transformed variables. The transformation is called a one-parameter Lie point transformation if and only if the transformed variables are given by:

$$
\begin{aligned}
\overline{y_{i}} & =y_{i}+\xi_{y_{i}}\left(y_{1}, \ldots, y_{n}, x_{1}, \ldots, x_{n}, t, \phi\right) \epsilon, \\
\overline{x_{i}} & =x_{i}+\xi_{x_{i}}\left(y_{1}, \ldots, y_{n}, x_{1}, \ldots, x_{n}, t, \phi\right) \epsilon, \\
\bar{t} & =t+\xi_{t}\left(y_{1}, \ldots, y_{n}, x_{1}, \ldots, x_{n}, t, \phi\right) \epsilon, \\
\bar{\phi} & =\phi+\eta_{\phi}\left(y_{1}, \ldots, y_{n}, x_{1}, \ldots, x_{n}, t, \phi\right) \epsilon
\end{aligned}
$$$$
i=1, \ldots, n,
$$$$
i=1, \ldots, n \text {, }
$$ 
In a one-parameter Lie point transformation, the derivatives of $\phi$ are not transformed separately. The transformations of $\underset{1}{\phi}, \underset{2}{\phi}, \ldots, \underset{q}{\phi}$ are calculated as functions of $\xi_{y_{1}}, \ldots, \xi_{y_{n}}, \xi_{x_{1}}, \ldots, \xi_{x_{n}}, \xi_{t}, \eta_{\phi}$. We write:

$$
\begin{aligned}
& \bar{\phi}_{, \bar{t}}=\phi_{, t}+\zeta_{; t}\left(y_{1}, \ldots, y_{n}, x_{1}, \ldots, x_{n}, t, \phi, \phi\right) \epsilon, \\
& \bar{\phi}_{, \bar{y}_{i}}=\phi_{, y_{i}}+\zeta_{; y_{i}}\left(y_{1}, \ldots, y_{n}, x_{1}, \ldots, x_{n}, t, \phi, \underset{1}{\phi} \epsilon, \quad i=1, \ldots, n,\right.
\end{aligned}
$$

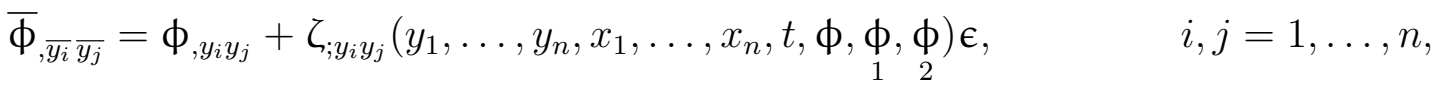

The functions $\xi_{y_{i}}, \xi_{x_{i}}, \xi_{t}, \eta_{\phi}, \zeta_{; \ldots}$ are called infinitesimals. Notice that we separate indices of $\zeta$ by a semicolon to distinguish them from derivatives. The transformations are expanded in a Taylor series:

$$
(T \bar{f})_{\epsilon_{0}}(\epsilon):=\left.\sum_{k=0}^{\infty} \frac{1}{k !} \frac{\partial^{k} \bar{f}}{\partial \epsilon^{k}}\right|_{\epsilon=\epsilon_{0}}\left(\epsilon-\epsilon_{0}\right)^{k}
$$

about $\epsilon_{0}=0$; hence, the infinitesimals are defined by:

$$
\begin{aligned}
\xi_{y_{i}} & :=\left.\frac{\partial \overline{y_{i}}}{\partial \epsilon}\right|_{\epsilon=0}, \quad \xi_{x_{i}}:=\left.\frac{\partial \overline{x_{i}}}{\partial \epsilon}\right|_{\epsilon=0}, \quad \xi_{t}:=\left.\frac{\partial \bar{t}}{\partial \epsilon}\right|_{\epsilon=0}, \quad \eta_{\phi}:=\left.\frac{\partial \bar{\phi}}{\partial \epsilon}\right|_{\epsilon=0}, \\
\zeta_{; t} & :=\left.\frac{\partial \bar{\phi}_{, \bar{t}}}{\partial \epsilon}\right|_{\epsilon=0}, \quad \zeta_{; y_{i}}:=\left.\frac{\partial \bar{\phi}_{\overline{y_{i}}}}{\partial \epsilon}\right|_{\epsilon=0}, \quad \cdots
\end{aligned}
$$

We extend this definition to FDEs.

Definition 5 (One-parameter Lie point transformations of FDEs). Consider the FDE

$$
F\left(\left[y\left(x^{\prime}\right)\right], x, t, \underset{1}{\phi} \underset{2}{\phi} \underset{3}{\phi}\right)=0
$$

and let:

$$
\begin{aligned}
\overline{y(z) \mathrm{d} z} & =\overline{y(z) \mathrm{d} z}\left(\left[y\left(x^{\prime}\right)\right], z, x, t, \phi, \epsilon\right), & & x^{\prime}, z \in G, \\
\bar{x} & =\bar{x}\left(\left[y\left(x^{\prime}\right)\right], x, t, \phi, \epsilon\right), & & x^{\prime} \in G, \\
\bar{t} & =\bar{t}\left(\left[y\left(x^{\prime}\right)\right], x, t, \phi, \epsilon\right), & & x^{\prime} \in G, \\
\bar{\phi} & =\bar{\phi}\left(\left[y\left(x^{\prime}\right)\right], x, t, \phi, \epsilon\right), & & x^{\prime} \in G,
\end{aligned}
$$

be the transformed variables where $G \subseteq \mathbb{R}$ is an uncountable set. The transformation is called the one-parameter Lie point transformation if and only if the transformed variables are given by:

$$
\begin{aligned}
\overline{y(z) \mathrm{d} z} & =y(z) \mathrm{d} z+\xi_{y(z)} \mathrm{d} z\left(\left[y\left(x^{\prime}\right)\right], z, x, t, \phi\right) \epsilon, & & x^{\prime}, z \in G, \\
\bar{x} & =x+\xi_{x}\left(\left[y\left(x^{\prime}\right)\right], x, t, \phi\right) \epsilon, & & x^{\prime} \in G, \\
\bar{t} & =t+\xi_{t}\left(\left[y\left(x^{\prime}\right)\right], x, t, \phi\right) \epsilon, & & x^{\prime} \in G, \\
\bar{\phi} & =\phi+\eta_{\phi}\left(\left[y\left(x^{\prime}\right)\right], x, t, \phi\right) \epsilon, & & x^{\prime} \in G
\end{aligned}
$$


Again, the derivatives of $\phi$ are not transformed separately. The transformations of $\phi, \phi, \phi$ are calculated as functionals depending on $\xi_{y(z)} \mathrm{d} z, \xi_{x}, \xi_{t}, \eta_{\phi}$. We write:

$$
\begin{array}{rlrl}
\bar{\phi}_{\overline{,}} & =\phi_{, t}+\zeta_{; t}\left(\left[y\left(x^{\prime}\right)\right], x, t, \phi, \underset{1}{\phi}\right) \epsilon, & x^{\prime} \in G, \\
\bar{\phi}_{, \overline{y\left(z_{1}\right)}} & =\phi_{, y\left(z_{1}\right)}+\zeta_{; y\left(z_{1}\right)}\left(\left[y\left(x^{\prime}\right)\right], z_{1}, x, t, \phi, \underset{1}{\phi}\right) \epsilon, & x^{\prime}, z_{1} \in G, \\
\bar{\phi}_{, y\left(z_{2}\right)} \frac{y_{\left(z_{1}\right)}}{}=\phi_{, y\left(z_{2}\right) y\left(z_{1}\right)}+\zeta_{; y\left(z_{2}\right) y\left(z_{1}\right)}\left(\left[y\left(x^{\prime}\right)\right], z_{1}, z_{2}, x, t, \underset{1}{\phi} \underset{2}{\phi}\right) \epsilon, & x^{\prime}, z_{1}, z_{2} \in G,
\end{array}
$$

The functionals $\xi_{y(z)} \mathrm{d} z, \xi_{x}, \xi_{t}, \eta_{\phi}, \zeta_{; t}, \ldots$ are called infinitesimals. Again, we separate indices of $\zeta$ by a semicolon to distinguish them from derivatives. The transformations are expanded in a Taylor series (9) about $\epsilon_{0}=0$; hence, the infinitesimals are defined by:

$$
\begin{aligned}
& \xi_{y(z)} \mathrm{d} z:=\left.\frac{\partial \overline{y(z) \mathrm{d} z}}{\partial \epsilon}\right|_{\epsilon=0}, \quad \xi_{x}:=\left.\frac{\partial \bar{x}}{\partial \epsilon}\right|_{\epsilon=0}, \quad \xi_{t}:=\left.\frac{\partial \bar{t}}{\partial \epsilon}\right|_{\epsilon=0}, \quad \eta_{\phi}:=\left.\frac{\partial \bar{\phi}}{\partial \epsilon}\right|_{\epsilon=0}, \\
& \zeta_{; t}:=\left.\frac{\partial \bar{\phi}_{, \bar{\epsilon}}}{\partial \epsilon}\right|_{\epsilon=0}, \quad \zeta_{; y(z)}:=\left.\frac{\partial \bar{\phi}, \overline{y(z)}}{\partial \epsilon}\right|_{\epsilon=0}, \quad \ldots
\end{aligned}
$$

Comparing the notion of one-parameter Lie point transformations of PDEs and of FDEs, one sees that the considered transformed functions $\overline{y_{i}}, \overline{x_{i}}, \bar{t}, \bar{\phi}$ are replaced by functionals $\overline{y(z) \mathrm{d} z}, \bar{x}, \bar{t}, \bar{\phi}$ depending on the infinite set $\left[y\left(x^{\prime}\right)\right]$. Notice that we do not transform $y(z)$, but $y(z) \mathrm{d} z$, as

$$
\int_{G} y(z) \mathrm{d} z \text { is the continuum analogon of } \sum_{i=1}^{n} y_{i}
$$

Following [14], the variable $y(z) \mathrm{d} z$ can be represented as a test series:

$$
y(z) \mathrm{d} z=\sum_{n=1}^{\infty} y_{n} h_{n}(z) \mathrm{d} z
$$

where $\left\{h_{n}(z)\right\}$ is a set of orthogonal functions. In this respect, the transformed variable $\overline{y(z) \mathrm{d} z}$ reads:

$$
\overline{y(z) \mathrm{d} z}=\sum_{n=1}^{\infty} \overline{y_{n}} h_{n}(\bar{z}) \mathrm{d} \bar{z}
$$

A possible approach to extending Lie group analysis would be to account for the transformations of $y_{n}$ and $z$, separately. However, due to the presence of functional derivatives in Equation (8), i.e., derivatives with respect to $y(z) \mathrm{d} z$, we prefer to consider $y(z) \mathrm{d} z$ as a variable to be transformed. Still, the decomposition (10) will be taken into account in the definitions of infinitesimals in Section 2.3.

An additional option would be to transform $y(z)$ instead of $y(z) \mathrm{d} z$. Then, one has to take into consideration the transformation of $\mathrm{d} z$. There are two ways that this could be done ( $f$. Section 3.1); however, we do not pursue those methods here. In order to be consistent, the infinitesimal associated with the transformation $\overline{y(z) \mathrm{d} z}$ is called $\xi_{y(z)} \mathrm{d} z$ instead of $\xi_{y(z)}$. The infinitesimal $\xi_{y(z)} \mathrm{d} z$ has to depend explicitly on $z$, as it defines a new variable for each $z \in G$. Analogous considerations hold true for the transformations of the functional derivatives of the dependent variable $\bar{\phi}_{, \ldots}$ and their infinitesimals $\zeta_{; \ldots}$. 


\subsection{Differential Operators}

In the classical Lie symmetry analysis, the variables $\left(y_{1}, \ldots, y_{n}, x_{1}, \ldots, x_{n}, t, \underset{1}{\phi}, \underset{2}{\phi}, \underset{3}{\phi}\right)$ are treated as independent variables. Hence, in the extended Lie symmetry analysis, we treat the variables $\left(\left[y\left(x^{\prime}\right)\right], x, t, \phi, \underset{1}{\phi}, \underset{2}{\phi}, \phi\right)$ as independent variables. The Hopf functional $\phi$ does not depend explicitly on $x$; hence, all derivatives of $\phi$ with respect to $x$ vanish, except when $\phi$ is derived first with respect to $y\left(x^{\prime}\right)$ and then with respect to $x$. Neglecting the vanishing summands and considering the non-commutativity:

$$
\frac{\partial^{2}}{\partial x \partial y(x) \mathrm{d} x} \neq \frac{\partial^{2}}{\partial y(x) \mathrm{d} x \partial x}
$$

we can define:

Definition 6 (Differential operators for Hopf FDEs). For a Hopf FDE of third order:

$$
F\left(\left[y\left(x^{\prime}\right)\right], x, t, \phi \underset{1}{\phi} \underset{2}{\phi} \underset{3}{\phi}\right)=0
$$

we introduce:

$$
\begin{aligned}
\frac{\mathcal{D}}{\mathcal{D} t}= & \frac{\partial}{\partial t}+\phi_{, t} \frac{\partial}{\partial \phi}+\phi_{, t t} \frac{\partial}{\partial \phi_{, t}}+\int_{G} \phi_{, t y\left(x^{\prime}\right)} \mathrm{d} x^{\prime} \frac{\partial}{\partial \phi_{, y\left(x^{\prime}\right)} \mathrm{d} x^{\prime}}+\phi_{, t t t} \frac{\partial}{\partial \phi_{, t t}} \\
& +\int_{G} \phi_{, t t y\left(x^{\prime}\right)} \mathrm{d} x^{\prime} \frac{\partial}{\partial \phi_{, t y\left(x^{\prime}\right)} \mathrm{d} x^{\prime}}+\int_{G} \phi_{, t x y\left(x^{\prime}\right)} \mathrm{d} x^{\prime} \frac{\partial}{\partial \phi_{, x y\left(x^{\prime}\right)} \mathrm{d} x^{\prime}} \\
& +\int_{G} \int_{G} \phi_{, t y\left(x^{\prime \prime}\right) y\left(x^{\prime}\right)} \mathrm{d} x^{\prime} \mathrm{d} x^{\prime \prime} \frac{\partial}{\partial \phi_{, y\left(x^{\prime \prime}\right) y\left(x^{\prime}\right)} \mathrm{d} x^{\prime} \mathrm{d} x^{\prime \prime}}, \\
\frac{\mathcal{D}}{\mathcal{D} x}= & \frac{\partial}{\partial x}+\int_{G} \phi_{, x y\left(x^{\prime}\right)} \mathrm{d} x^{\prime} \frac{\partial}{\partial \phi_{, y\left(x^{\prime}\right)} \mathrm{d} x^{\prime}}+\int_{G} \phi_{, x t y\left(x^{\prime}\right)} \mathrm{d} x^{\prime} \frac{\partial}{\partial \phi_{, t y\left(x^{\prime}\right)} \mathrm{d} x^{\prime}} \\
& +\int_{G} \phi_{, x x y\left(x^{\prime}\right)} \mathrm{d} x^{\prime} \frac{\partial}{\partial \phi_{, x y\left(x^{\prime}\right)} \mathrm{d} x^{\prime}}+\int_{G} \int_{G} \phi_{, x y\left(x^{\prime \prime}\right) y\left(x^{\prime}\right)} \mathrm{d} x^{\prime} \mathrm{d} x^{\prime \prime} \frac{\partial}{\partial \phi_{, y\left(x^{\prime \prime}\right) y\left(x^{\prime}\right)} \mathrm{d} x^{\prime} \mathrm{d} x^{\prime \prime}}, \\
\mathcal{D} y(z) \mathrm{d} z= & \frac{\partial}{\partial y(z) \mathrm{d} z}+\phi_{, y(z)} \frac{\partial}{\partial \phi}+\phi_{, y(z) t} \frac{\partial}{\partial \phi_{, t}}+\int_{G} \phi_{, y(z) y\left(x^{\prime}\right)} \mathrm{d} x^{\prime} \frac{\partial}{\partial \phi_{, y\left(x^{\prime}\right)} \mathrm{d} x^{\prime}}+\phi_{, y(z) t t} \frac{\partial}{\partial \phi_{, t t}} \\
& +\int_{G} \phi_{, y(z) t y\left(x^{\prime}\right)} \mathrm{d} x^{\prime} \frac{\partial}{\partial \phi_{, t y\left(x^{\prime}\right)} \mathrm{d} x^{\prime}}+\int_{G} \phi_{, y(z) x y\left(x^{\prime}\right)} \mathrm{d} x^{\prime} \frac{\partial}{\partial \phi_{, x y\left(x^{\prime}\right)} \mathrm{d} x^{\prime}} \\
& +\int_{G} \int_{G} \phi_{, y(z) y\left(x^{\prime \prime}\right) y\left(x^{\prime}\right)} \mathrm{d} x^{\prime} \mathrm{d} x^{\prime \prime} \frac{\partial}{\partial \phi_{, y\left(x^{\prime \prime}\right) y\left(x^{\prime}\right)} \mathrm{d} x^{\prime} \mathrm{d} x^{\prime \prime}}
\end{aligned}
$$

\subsection{Infinitesimals}

By means of the differential operators presented in Definition 6, we can calculate the infinitesimals $\zeta_{; \ldots}$ as functionals of the infinitesimals $\xi_{y(z)} \mathrm{d} z, \xi_{x}, \xi_{t}, \eta_{\phi}$. For the viscous Hopf-Burgers FDE, we need the three infinitesimals $\zeta_{; t}, \zeta_{; x y(x) y(x)}$ and $\zeta_{; x x y(x)}$.

- In order to calculate $\zeta_{; t}$, we differentiate the transformed Hopf functional $\bar{\phi}$ with respect to $t$, taking into account the decomposition (10):

$$
\frac{\mathcal{D} \bar{\phi}}{\mathcal{D} t}=\frac{\mathcal{D} \bar{\phi}}{\mathcal{D} \bar{t}} \frac{\mathcal{D} \overline{\mathcal{D}} t}{\mathcal{D}}+\frac{\mathcal{D} \bar{\phi}}{\mathcal{D} \bar{x}} \frac{\mathcal{D} \bar{x}}{\mathcal{D} t}+\int_{\bar{G}} \frac{\mathcal{D} \bar{\phi}}{\overline{\mathcal{D} y\left(x^{\prime}\right) \mathrm{d} x^{\prime}}} \sum_{n=1}^{\infty} \frac{\mathcal{D} \overline{y_{n}}}{\mathcal{D} t} h_{n}\left(\overline{x^{\prime}}\right) \mathrm{d} \overline{x^{\prime}}
$$


Hence, in the definition above, we account for the fact that $t, x$ and the infinite set $\left\{y_{n}\right\}$ are the independent variables. Still, as argued in Section 2.1, in the extended Lie group analysis, we transform the continuum variable $y(x) \mathrm{d} x$; hence, we will express the last RHS term in Equation (12) in terms of $y(x) \mathrm{d} x$. This step is crucial for further successful recovery of the symmetries of the Burgers equation. Using (10) again, the time differential operator applied to $\overline{y\left(x^{\prime}\right) \mathrm{d} x^{\prime}}$ can be decomposed into:

$$
\frac{\mathcal{D} \overline{y(x) \mathrm{d} x}}{\mathcal{D} t}=\sum_{n=1}^{\infty} \frac{\mathcal{D} \overline{y_{n}}}{\mathcal{D} t} h_{n}(\bar{x}) \mathrm{d} \bar{x}+\sum_{n=1}^{\infty} y_{n} \frac{\mathcal{D} h_{n}(\bar{x}) \mathrm{d} \bar{x}}{\mathcal{D} t}
$$

The second RHS term can be rewritten as:

$$
\sum_{n=1}^{\infty} \frac{\mathcal{D} \overline{y_{n}}}{\mathcal{D} t} h_{n}(\bar{x}) \mathrm{d} \bar{x}=\frac{\mathcal{D} \overline{y(x) \mathrm{d} x}}{\mathcal{D} t}-\frac{\mathcal{D} \overline{y(x) \mathrm{d} x}}{\mathcal{D} \bar{x}} \frac{\mathcal{D} \bar{x}}{\mathcal{D} t}
$$

and substituted into Equation (12). Taking into account the fact that $\bar{\phi}$ does not depend explicitly on $\bar{x}$, from Equation (12), we obtain:

$$
\frac{\mathcal{D} \bar{\phi}}{\mathcal{D} t}=\frac{\mathcal{D} \bar{\phi}}{\mathcal{D} \bar{t}} \frac{\mathcal{D} \overline{\mathcal{D}} t}{\mathcal{L}}+\int_{\bar{G}} \frac{\mathcal{D} \bar{\phi}}{\overline{\mathcal{D} y\left(x^{\prime}\right) \mathrm{d} x^{\prime}}} \frac{\mathcal{D} \overline{y\left(x^{\prime}\right) \mathrm{d} x^{\prime}}}{\mathcal{D} t}-\int_{\bar{G}} \frac{\mathcal{D} \bar{\phi}}{\overline{\mathcal{D} y\left(x^{\prime}\right) \mathrm{d} x^{\prime}}} \frac{\mathcal{D} \overline{x^{\prime}}}{\mathcal{D} t} \frac{\overline{\mathcal{D} y\left(x^{\prime}\right) \mathrm{d} x^{\prime}}}{\mathcal{D} \overline{x^{\prime}}}
$$

We integrate the last term by parts and assume that $\phi$ and its derivatives are zero at the boundaries. This step is necessary, as we further want to introduce Lie point transformations ( $c f$. Definition 5) for the quantities in the integral. We finally obtain:

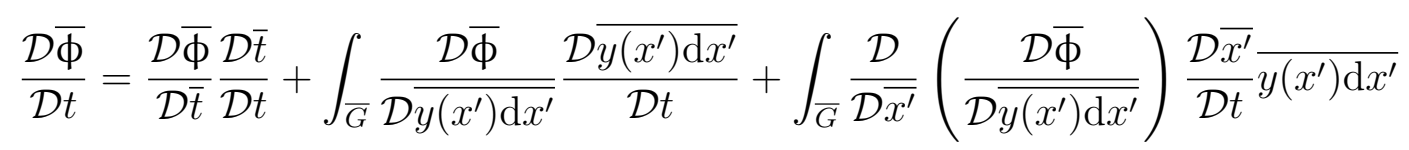

With the one-parameter Lie point transformations ( $c f$. Definition 5), Equation (16) reads:

$$
\begin{aligned}
\frac{\mathcal{D}\left(\phi+\epsilon \eta_{\phi}\right)}{\mathcal{D} t} & =\left(\phi_{, t}+\epsilon \zeta_{; t}\right) \frac{\mathcal{D}\left(t+\epsilon \xi_{t}\right)}{\mathcal{D} t}+\int_{G}\left(\phi_{, y\left(x^{\prime}\right)}+\epsilon \zeta_{; y\left(x^{\prime}\right)}\right) \frac{\mathcal{D}\left(y\left(x^{\prime}\right) \mathrm{d} x^{\prime}+\epsilon \xi_{y\left(x^{\prime}\right)} \mathrm{d} x^{\prime}\right)}{\mathcal{D} t} \\
& +\int_{G}\left(\phi_{, x^{\prime} y\left(x^{\prime}\right)}+\epsilon \zeta_{; x^{\prime} y\left(x^{\prime}\right)}\right) \frac{\mathcal{D}\left(x^{\prime}+\epsilon \xi_{x^{\prime}}\right)}{\mathcal{D} t}\left(y\left(x^{\prime}\right) \mathrm{d} x^{\prime}+\epsilon \xi_{y\left(x^{\prime}\right)} \mathrm{d} x^{\prime}\right)
\end{aligned}
$$

Evaluating this equation in $\mathcal{O}(1)$, we get

$$
\frac{\mathcal{D} \phi}{\mathcal{D} t}=\phi_{, t}
$$

Using this, evaluating in $\mathcal{O}(\epsilon)$ leads to:

$$
\frac{\mathcal{D} \eta_{\phi}}{\mathcal{D} t}=\phi_{, t} \frac{\mathcal{D} \xi_{t}}{\mathcal{D} t}+\zeta_{; t}+\int_{G} \phi_{, y\left(x^{\prime}\right)} \frac{\mathcal{D} \xi_{y\left(x^{\prime}\right)} \mathrm{d} x^{\prime}}{\mathcal{D} t}+\int_{G} \phi_{, x^{\prime} y\left(x^{\prime}\right)} \frac{\mathcal{D} \xi_{x^{\prime}}}{\mathcal{D} t} y\left(x^{\prime}\right) \mathrm{d} x^{\prime}
$$

which constitutes an equation for $\zeta_{;}$:

$$
\zeta_{; t}=\frac{\mathcal{D} \eta_{\phi}}{\mathcal{D} t}-\phi_{, t} \frac{\mathcal{D} \xi_{t}}{\mathcal{D} t}-\int_{G} \phi_{, y\left(x^{\prime}\right)} \frac{\mathcal{D} \xi_{y\left(x^{\prime}\right)} \mathrm{d} x^{\prime}}{\mathcal{D} t}-\int_{G} \phi_{, x^{\prime} y\left(x^{\prime}\right)} \frac{\mathcal{D} \xi_{x^{\prime}}}{\mathcal{D} t} y\left(x^{\prime}\right) \mathrm{d} x^{\prime}
$$


Since the appearing infinitesimals $\xi_{y\left(x^{\prime}\right)} \mathrm{d} x^{\prime}, \xi_{t}, \eta_{\phi}$ do not depend on derivatives of $\phi$, we immediately get:

$$
\begin{aligned}
\zeta_{; t} & =\frac{\partial \eta_{\phi}}{\partial t}+\phi_{, t}\left(\frac{\partial \eta_{\phi}}{\partial \phi}-\frac{\partial \xi_{t}}{\partial t}\right)-\left(\phi_{, t}\right)^{2} \frac{\partial \xi_{t}}{\partial \phi}-\int_{G} \phi_{, y\left(x^{\prime}\right)} \frac{\partial \xi_{y\left(x^{\prime}\right)} \mathrm{d} x^{\prime}}{\partial t}-\int_{G} \phi_{, y\left(x^{\prime}\right)} \phi_{, t} \frac{\partial \xi_{y\left(x^{\prime}\right)} \mathrm{d} x^{\prime}}{\partial \phi} \\
& -\int_{G} \phi_{, x^{\prime} y\left(x^{\prime}\right)} \frac{\partial \xi_{x^{\prime}}}{\partial t} y\left(x^{\prime}\right) \mathrm{d} x^{\prime}-\int_{G} \phi_{, x^{\prime} y\left(x^{\prime}\right)} \phi_{, t} \frac{\partial \xi_{x^{\prime}}}{\partial \phi} y\left(x^{\prime}\right) \mathrm{d} x^{\prime}
\end{aligned}
$$

- In order to calculate $\zeta_{; y(x)}$, we differentiate $\bar{\phi}$ with respect to $y(x)$. An analogouscalculation leads to:

$$
\begin{aligned}
\zeta_{; y(x)}= & \frac{\mathcal{D} \eta_{\phi}}{\mathcal{D} y(x) \mathrm{d} x}-\phi_{, t} \frac{\mathcal{D} \xi_{t}}{\mathcal{D} y(x) \mathrm{d} x}-\int_{G} \phi_{, y\left(x^{\prime}\right)} \frac{\mathcal{D} \xi_{y\left(x^{\prime}\right)} \mathrm{d} x^{\prime}}{\mathcal{D} y(x) \mathrm{d} x}-\int \phi_{, x^{\prime} y\left(x^{\prime}\right)} \frac{\mathcal{D} \xi_{x^{\prime}}}{\mathcal{D} y(x) \mathrm{d} x} y\left(x^{\prime}\right) \mathrm{d} x^{\prime} \\
= & \frac{\partial \eta_{\phi}}{\partial y(x) \mathrm{d} x}+\phi_{, y(x)} \frac{\partial \eta_{\phi}}{\partial \phi}-\phi_{, t} \frac{\partial \xi_{t}}{\partial y(x) \mathrm{d} x}-\phi_{, t} \phi_{, y(x)} \frac{\partial \xi_{t}}{\partial \phi}-\int_{G} \phi_{, y\left(x^{\prime}\right)} \frac{\partial \xi_{y\left(x^{\prime}\right)} \mathrm{d} x^{\prime}}{\partial y(x) \mathrm{d} x} \\
& -\int_{G} \phi_{, y\left(x^{\prime}\right)} \phi_{, y(x)} \frac{\partial \xi_{y\left(x^{\prime}\right)} \mathrm{d} x^{\prime}}{\partial \phi}-\int \phi_{, x^{\prime} y\left(x^{\prime}\right)} \frac{\partial \xi_{x^{\prime}}}{\partial y(x) \mathrm{d} x} y\left(x^{\prime}\right) \mathrm{d} x^{\prime}-\int \phi_{, x^{\prime} y\left(x^{\prime}\right)} \phi_{, y(x)} \frac{\mathrm{d} \xi_{x^{\prime}}}{\partial \phi} y\left(x^{\prime}\right) \mathrm{d} x^{\prime}
\end{aligned}
$$

In order to calculate $\zeta_{; y(x) y(x)}$, we differentiate $\bar{\phi}, \overline{y(x)}$ with respect to $y(x)$. As $\bar{\phi} \frac{}{y(x)}$ does depend on $\bar{x}$, we have:

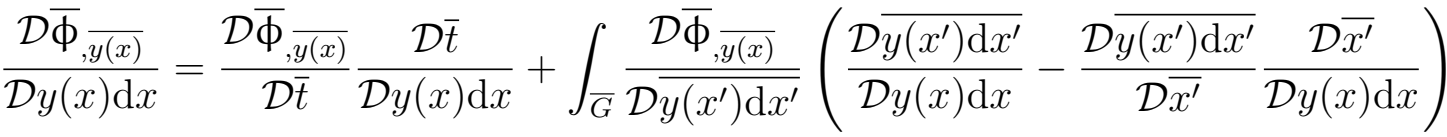

$$
\begin{aligned}
& +\frac{\mathcal{D} \bar{\phi}, \overline{y(x)}}{\mathcal{D} \bar{x}} \frac{\mathcal{D} \bar{x}}{\mathcal{D} y(x) \mathrm{d} x} \\
& =\bar{\phi}_{, \bar{t}} \overline{y(x)} \frac{\mathcal{D} \bar{t}}{\mathcal{D} y(x) \mathrm{d} x}+\int_{\bar{G}} \bar{\phi} \frac{\overline{\mathcal{y}\left(x^{\prime}\right)}}{y(x)} \frac{\overline{\mathcal{D}\left(x^{\prime}\right) \mathrm{d} x^{\prime}}}{\mathcal{D} y(x) \mathrm{d} x}+\int_{G} \bar{\phi}_{, \bar{x}^{\prime} y\left(x^{\prime}\right)} \frac{\overline{\mathcal{D}} \overline{x^{\prime}}}{\overline{\mathcal{D} y} y(x) \mathrm{d} x} \overline{y\left(x^{\prime}\right) \mathrm{d} x^{\prime}} \\
& +\bar{\phi}_{, \bar{x}} \frac{\mathcal{D} \bar{x}}{\bar{y}(x)} \frac{\mathrm{D} y(x) \mathrm{d} x}{2}
\end{aligned}
$$

If we insert the one-parameter Lie point transformations ( $c f$. Definition 5), we get:

$$
\begin{aligned}
\zeta_{; y(x) y(x)} & =\frac{\mathcal{D} \zeta_{; y(x)}}{\mathcal{D} y(x) \mathrm{d} x}-\phi_{, t y(x)} \frac{\mathcal{D} \xi_{t}}{\mathcal{D} y(x) \mathrm{d} x}-\int_{G} \phi_{, y\left(x^{\prime}\right) y(x)} \frac{\mathcal{D} \xi_{y\left(x^{\prime}\right)} \mathrm{d} x^{\prime}}{\mathcal{D} y(x) \mathrm{d} x}-\phi_{, x y(x)} \frac{\mathcal{D} \xi_{x}}{\mathcal{D} y(x) \mathrm{d} x} \\
& -\int_{G} \phi_{, x^{\prime} y\left(x^{\prime}\right) y(x)} \frac{\mathcal{D} \xi_{x^{\prime}}}{\mathcal{D} y(x) \mathrm{d} x} y\left(x^{\prime}\right) \mathrm{d} x^{\prime}
\end{aligned}
$$

In order to get $\zeta_{; x y(x) y(x)}$, we differentiate $\bar{\phi} \overline{, y(x)} \frac{}{y(x)}$ with respect to $x$ :

$$
\begin{aligned}
& \frac{\mathcal{D} \bar{\phi} \overline{, y(x)} \overline{y(x)}}{\mathcal{D} x}=\frac{\mathcal{D} \bar{\phi}, \overline{y(x)} \overline{y(x)}}{\mathcal{D} \bar{t}} \frac{\mathcal{D} \bar{t}}{\mathcal{D} x}+\int_{\bar{G}} \frac{\mathcal{D} \bar{\phi} \overline{y(x)} \overline{y(x)}}{\mathcal{D} \overline{y\left(x^{\prime}\right) \mathrm{d} x^{\prime}}} \frac{\mathcal{D} \overline{y\left(x^{\prime}\right) \mathrm{d} x^{\prime}}}{\mathcal{D} x}+\frac{\mathcal{D} \bar{\phi}, \overline{y(x)} \overline{y(x)}}{\mathcal{D} \bar{x}} \frac{\mathcal{D} \bar{x}}{\mathcal{D} x}
\end{aligned}
$$

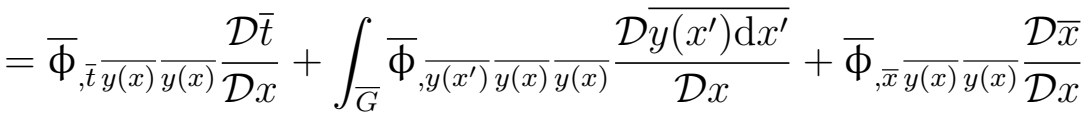

If we insert the one-parameter Lie point transformations ( $c f$. Definition 5), we get:

$$
\zeta_{; x y(x) y(x)}=\frac{\mathcal{D} \zeta_{; y(x) y(x)}}{\mathcal{D} x}-\phi_{, t y(x) y(x)} \frac{\mathcal{D} \xi_{t}}{\mathcal{D} x}-\int_{G} \phi_{, y\left(x^{\prime}\right) y(x) y(x)} \frac{\mathcal{D} \xi_{y\left(x^{\prime}\right)} \mathrm{d} x^{\prime}}{\mathcal{D} x}-\phi_{, x y(x) y(x)} \frac{\mathcal{D} \xi_{x}}{\mathcal{D} x}
$$


- In order to calculate $\zeta_{; x y(x)}$, we differentiate $\bar{\phi}_{, y(x)}$ with respect to $x$. We have:

$$
\begin{aligned}
& \frac{\mathcal{D} \bar{\phi}, \overline{y(x)}}{\mathcal{D} x}=\frac{\mathcal{D} \bar{\phi}, \overline{y(x)}}{\mathcal{D} \bar{t}} \frac{\mathcal{D} \bar{t}}{\mathcal{D} x}+\int_{\bar{G}} \frac{\mathcal{D} \bar{\phi}, \overline{y(x)}}{\mathcal{D} \overline{y\left(x^{\prime}\right) \mathrm{d} x^{\prime}}} \frac{\mathcal{D} \overline{y\left(x^{\prime}\right) \mathrm{d} x^{\prime}}}{\mathcal{D} x}+\frac{\mathcal{D} \bar{\phi}, \overline{y(x)}}{\mathcal{D} \bar{x}} \frac{\mathcal{D} \bar{x}}{\mathcal{D} x} \\
& =\bar{\phi}_{\overline{,} \bar{y}} \frac{\mathcal{D} \bar{t} \bar{t}}{\mathcal{D} x}+\int_{\bar{G}} \bar{\phi}_{, \overline{y\left(x^{\prime}\right)}} \frac{\mathcal{D} y(x)}{\overline{y\left(x^{\prime}\right) \mathrm{d} x^{\prime}}}+\bar{\phi}_{, \bar{x}} \frac{\mathcal{D} \overline{y(x)}}{\mathcal{D} x}
\end{aligned}
$$

If we insert the one-parameter Lie point transformations ( $c f$. Definition 5), we get:

$$
\zeta_{; x y(x)}=\frac{\mathcal{D} \zeta_{; y(x)}}{\mathcal{D} x}-\phi_{, t y(x)} \frac{\mathcal{D} \xi_{t}}{\mathcal{D} x}-\int_{G} \phi_{, y\left(x^{\prime}\right) y(x)} \frac{\mathcal{D} \xi_{y\left(x^{\prime}\right)} \mathrm{d} x^{\prime}}{\mathcal{D} x}-\phi_{, x y(x)} \frac{\mathcal{D} \xi_{x}}{\mathcal{D} x}
$$

In order to get $\zeta_{; x x y(x)}$, we differentiate $\bar{\phi}_{, \bar{x}} \overline{y(x)}$ with respect to $x$ :

$$
\begin{aligned}
\frac{\mathcal{D} \bar{\phi}_{\bar{x}} \overline{y(x)}}{\mathcal{D} x} & =\frac{\mathcal{D} \bar{\phi}_{\bar{x}} \overline{y(x)}}{\mathcal{D} \bar{t}} \frac{\mathcal{D} \bar{t}}{\mathcal{D} x}+\int_{\bar{G}} \frac{\mathcal{D} \bar{\phi}_{, \bar{x}} \overline{\mathcal{y}(x)}}{\overline{\mathcal{D} y\left(x^{\prime}\right) \mathrm{d} x^{\prime}}} \frac{\overline{\mathcal{D} y\left(x^{\prime}\right) \mathrm{d} x^{\prime}}}{\mathcal{D} x}+\frac{\mathcal{D} \bar{\phi}_{\bar{x}} \overline{y(x)}}{\mathcal{D} \bar{x}} \frac{\mathcal{D} \bar{x}}{\mathcal{D} x} \\
& =\bar{\phi}_{, \bar{t} \bar{x} \overline{y(x)}} \frac{\mathcal{D} \overline{\mathcal{D}}}{\mathcal{D} x}+\int_{\bar{G}} \bar{\phi}_{, \overline{y\left(x^{\prime}\right)}} \bar{x} \overline{y(x)} \frac{\mathcal{D} y\left(x^{\prime}\right) \mathrm{d} x^{\prime}}{\mathcal{D} x}+\bar{\phi}_{, \bar{x} \bar{x} \overline{y(x)}} \frac{\mathcal{D} \overline{\mathcal{D}} x}{}
\end{aligned}
$$

If we insert the one-parameter Lie point transformations ( $c f$. Definition 5), we get:

$$
\zeta_{; x x y(x)}=\frac{\mathcal{D} \zeta_{; x y(x)}}{\mathcal{D} x}-\phi_{, t x y(x)} \frac{\mathcal{D} \xi_{t}}{\mathcal{D} x}-\int_{G} \phi_{, y\left(x^{\prime}\right) x y(x)} \frac{\mathcal{D} \xi_{y\left(x^{\prime}\right)} \mathrm{d} x^{\prime}}{\mathcal{D} x}-\phi_{, x x y(x)} \frac{\mathcal{D} \xi_{x}}{\mathcal{D} x}
$$

Applying the differential operators introduced in Definition 6, one can represent the infinitesimals (19) and (20) as sums of partial and functional derivatives of $\xi_{y(z)} \mathrm{d} z, \xi_{x}, \xi_{t}, \eta_{\phi} ; c f$. Appendix.

\subsection{Infinitesimal Generator and the Determining System of Equations for the Infinitesimals}

Let $F\left(\left[\overline{y\left(x^{\prime}\right)}\right], \bar{x}, \bar{t}, \bar{\phi}, \bar{\phi}, \underset{2}{\phi}, \bar{\phi}\right)=0$ be a transformed FDE. Consider $F$ as a function depending on the group parameter $\epsilon$, and expand $F$ in a Taylor series (9) about $\epsilon_{0}=0$, i.e., consider the equation:

$$
\left.F\right|_{\epsilon=0}+\left.\frac{\partial F}{\partial \epsilon}\right|_{\epsilon=0} \epsilon+\mathcal{O}\left(\epsilon^{2}\right)=0
$$

We calculate:

$$
\begin{aligned}
\left.\frac{\partial F}{\partial \epsilon}\right|_{\epsilon=0}= & {\left[\int_{G} \xi_{y\left(x^{\prime}\right)} \mathrm{d} x^{\prime} \frac{\partial}{\partial y\left(x^{\prime}\right) \mathrm{d} x^{\prime}}+\xi_{x} \frac{\partial}{\partial x}+\xi_{t} \frac{\partial}{\partial t}+\eta_{\phi} \frac{\partial}{\partial \phi}\right.} \\
& \left.+\zeta_{; t} \frac{\partial}{\partial \phi_{, t}}+\int_{G} \zeta_{; y\left(x^{\prime}\right)} \mathrm{d} x^{\prime} \frac{\partial}{\partial \phi_{, y\left(x^{\prime}\right)} \mathrm{d} x^{\prime}}+\ldots\right] F
\end{aligned}
$$

and define:

Definition 7 (Infinitesimal generator and its prolongation).

- The differential operator:

$$
X:=\int_{G} \xi_{y\left(x^{\prime}\right)} \mathrm{d} x^{\prime} \frac{\partial}{\partial y\left(x^{\prime}\right) \mathrm{d} x^{\prime}}+\xi_{x} \frac{\partial}{\partial x}+\xi_{t} \frac{\partial}{\partial t}+\eta_{\phi} \frac{\partial}{\partial \phi}
$$

is called the infinitesimal generator or simply the generator. 
- The differential operator:

$$
\begin{aligned}
X^{(3)}:= & X+\zeta_{; t} \frac{\partial}{\partial \phi_{, t}}+\int_{G} \zeta_{; y\left(x^{\prime}\right)} \mathrm{d} x^{\prime} \frac{\delta}{\delta \phi_{, y\left(x^{\prime}\right)}}+\zeta_{; t t} \frac{\partial}{\partial \phi_{, t t}}+\int_{G} \zeta_{; t y\left(x^{\prime}\right)} \mathrm{d} x^{\prime} \frac{\delta}{\delta \phi_{, t y\left(x^{\prime}\right)}} \\
& +\int_{G} \zeta_{; x y\left(x^{\prime}\right)} \mathrm{d} x^{\prime} \frac{\delta}{\delta \phi_{, x y\left(x^{\prime}\right)}}+\int_{G} \int_{G} \zeta_{; y\left(x^{\prime \prime}\right) y\left(x^{\prime}\right)} \mathrm{d} x^{\prime} \mathrm{d} x^{\prime \prime} \frac{\delta}{\delta \phi_{, y\left(x^{\prime \prime}\right) y\left(x^{\prime}\right)}} \\
& +\zeta_{; t t t} \frac{\partial}{\partial \phi_{, t t t}}+\int_{G} \zeta_{; t t y\left(x^{\prime}\right)} \mathrm{d} x^{\prime} \frac{\delta}{\delta \phi_{, t t y\left(x^{\prime}\right)}}+\int_{G} \int_{G} \zeta_{; t y\left(x^{\prime \prime}\right) y\left(x^{\prime}\right)} \mathrm{d} x^{\prime} \mathrm{d} x^{\prime \prime} \frac{\delta}{\delta \phi_{, t y\left(x^{\prime \prime}\right) y\left(x^{\prime}\right)}} \\
& +\int_{G} \zeta_{; t x y\left(x^{\prime}\right)} \mathrm{d} x^{\prime} \frac{\delta}{\delta \phi_{, t x y\left(x^{\prime}\right)}}+\int_{G} \int_{G} \int_{G} \zeta_{; y\left(x^{\prime \prime \prime}\right) y\left(x^{\prime \prime}\right) y\left(x^{\prime}\right)} \mathrm{d} x^{\prime} \mathrm{d} x^{\prime \prime} \mathrm{d} x^{\prime \prime \prime} \frac{\delta}{\delta \phi_{, y\left(x^{\prime \prime \prime}\right) y\left(x^{\prime \prime}\right) y\left(x^{\prime}\right)}} \\
& +\int_{G} \int_{G} \zeta_{; y\left(x^{\prime \prime}\right) x y\left(x^{\prime}\right)} \mathrm{d} x^{\prime} \mathrm{d} x^{\prime \prime} \frac{\delta}{\delta \phi_{, y\left(x^{\prime \prime}\right) x y\left(x^{\prime}\right)}}+\int_{G} \int_{G} \zeta_{; x y\left(x^{\prime \prime}\right) y\left(x^{\prime}\right)} \mathrm{d} x^{\prime} \mathrm{d} x^{\prime \prime} \frac{\delta}{\delta \phi_{, x y\left(x^{\prime \prime}\right) y\left(x^{\prime}\right)}} \\
& +\int_{G} \zeta_{; x x y\left(x^{\prime}\right)} \mathrm{d} x^{\prime} \frac{\delta}{\delta \phi_{, x x y\left(x^{\prime}\right)}}
\end{aligned}
$$

is called the prolongation of $X$.

Using this definition and employing $\left.F\right|_{\epsilon=0}=F$, Equation (21) reads:

$$
F+X^{(3)} F \epsilon+\mathcal{O}\left(\epsilon^{2}\right)=0
$$

This equation is fulfilled in $\mathcal{O}(\epsilon)$ if and only if:

$$
\left[X^{(3)} F\right]_{F=0}=0
$$

Equation (22) constitutes an overdetermined system of linear FDEs for the infinitesimals $\xi_{y(z)} \mathrm{d} z, \xi_{x}$, $\xi_{t}, \eta_{\phi}$. In order to formulate the system of equations, one has to insert the necessary infinitesimals, calculated in Section 2.3, into Equation (22). Since $\xi_{y(z)} \mathrm{d} z, \xi_{x}, \xi_{t}, \eta_{\phi}$ do not depend on the derivatives of $\phi$, all coefficients of all appearing derivatives of $\phi$ have to vanish, which leads to a system of linear FDEs.

\subsection{Global Transformations}

Knowing $\xi_{y(z)} \mathrm{d} z, \xi_{x}, \xi_{t}, \eta_{\phi}$, one obtains the global transformations using Lie's first theorem, $c f$. [13]:

Theorem 8 (Lie's first theorem). The global transformation can be obtained by solving the following initial value problems:

$$
\frac{\partial \overline{y(z) \mathrm{d} z}}{\partial \epsilon}=\xi_{\overline{y(z)}} \overline{\mathrm{d} z}, \quad \frac{\partial \bar{x}}{\partial \epsilon}=\xi_{\bar{x}}, \quad \frac{\partial \bar{t}}{\partial \epsilon}=\xi_{\bar{t}}, \quad \frac{\partial \bar{\phi}}{\partial \epsilon}=\eta_{\bar{\phi}}
$$

with the initial values:

$$
\overline{y(z) \mathrm{d} z}(\epsilon=0)=y(z) \mathrm{d} z, \quad \bar{x}(\epsilon=0)=x, \quad \bar{t}(\epsilon=0)=t, \quad \bar{\phi}(\epsilon=0)=\phi
$$




\section{Lie Symmetry Analysis of the Viscous Hopf-Burgers Functional Integro-Differential Equation}

\subsection{Three Different Approaches to Lie Symmetry Analysis}

As already mentioned in the Introduction, when working in the physical space, the main problem is to deal with the partial derivatives $\partial / \partial x$ and the transformation of the integration variable $x$. Different Lie symmetry analysis approaches for integro-differential equations have been proposed in the literature; see [15]. In the following, we present three methods that could possibly be applied in our case of functional equations with functional derivatives.

1. We transform $y(z)$ instead of $y(z) \mathrm{d} z$ and have to take into account the transformation of the integral term appearing in Hopf FDEs. In [13,16], Ibragimov suggests to use the fact that $X$ given by Definition 7 is equivalent to a canonical Lie-Bäcklund operator $\tilde{X}$, which does not contain the term $\xi_{x} \partial / \partial x$. This implies that $\tilde{X}$ is very suitable for the symmetry analysis of integro-differential equations. Hence, one might replace $X$ by $\tilde{X}$ and perform the extended Lie symmetry analysis on functional integro-differential equations.

2. We transform $y(z)$ instead of $y(z) \mathrm{d} z$ and consider the differential equation as an equation $F=0$, where $F$ depends on an integral term $I$ and an integral-free term $H$, i.e., $F(H, I)=0$. In order to get the correct determining system of equations for the infinitesimals, the transformation of $F$ is expanded in a two-dimensional Taylor series about $H$ and $I$. This method is presented by Zawistowski in [17].

In [18], it is shown that using Zawistowski's approach leads to results being equivalent to the results presented in Ibragimov's study and that the Lie algebra of symmetry group transformations spanned by the infinitesimal generators containing integral terms is solvable; $c f$. [17] for the Vlasov-Maxwell integro-differential equation and [18] for the Benney integro-differential equations. Zawistowski's approach leads to the following determining system of equations for the infinitesimals $\xi_{y(z)} \mathrm{d} z, \xi_{x}, \xi_{t}, \eta_{\phi}$ :

$$
\left[\mathcal{X}^{(3)} F-\int_{G} \frac{\partial \xi_{x^{\prime}}}{\partial x^{\prime}} f \mathrm{~d} x^{\prime}\right]_{F=0}=0
$$

One has to pay attention that the analogous formula for the transformed integral should be used during the calculation of $\zeta_{; t}, \zeta_{; x y(x) y(x)}$ and $\zeta_{; x x y(x)}$ (cf. Section 2.3) in order to determine the generator $\mathcal{X}^{(3)}$ given by Definition 7 , which, in our particular case of the equation with functional derivatives, makes this approach more complicated.

3. We transform $y(z) \mathrm{d} z$ instead of $y(z)$ and introduce a transformation of $x$. Then, we perform the extended Lie symmetry analysis on the viscous Hopf-Burgers FDE (8). Presently, this approach is successfully applied to (8), and we rediscover all known symmetries of the usual viscous Burgers Equation (6). The results and a discussion comparing the symmetries of the viscous Hopf-Burgers FDE and the symmetries of the viscous Burgers equation are presented in the following subsections. 


\subsection{Local Transformations of the Viscous Hopf-Burgers Functional Integro-Differential Equation}

3.2.1. Determining the System of Equations for the Infinitesimals

In this section, we perform the extended Lie symmetry analysis on the viscous Hopf-Burgers FDE:

$$
F=\phi_{, t}-\int_{G} y(x)\left(i \phi_{, x y(x) y(x)}+\boldsymbol{v} \phi_{, x x y(x)}\right) \mathrm{d} x=0
$$

We consider the one-parameter Lie point transformations given by Definition 5 .

In order to calculate the determining system of equations for the infinitesimals $\xi_{y(z)} \mathrm{d} z, \xi_{x}, \xi_{t}, \eta_{\phi}$, we consider Equation (22):

$$
\left[X^{(3)} F\right]_{F=0}=0
$$

The generator $X^{(3)}$ is given by Definition 7. The contributing summands of $X^{(3)}$ are given by:

$$
\begin{aligned}
X_{\text {modif }}^{(3)}= & \int_{G} \xi_{y\left(x^{\prime}\right)} \mathrm{d} x^{\prime} \frac{\partial}{\partial y\left(x^{\prime}\right) \mathrm{d} x^{\prime}}+\zeta_{; t} \frac{\partial}{\partial \phi_{, t}}+\int_{G} \int_{G} \zeta_{; x y\left(x^{\prime \prime}\right) y\left(x^{\prime}\right)} \mathrm{d} x^{\prime} \mathrm{d} x^{\prime \prime} \frac{\partial}{\partial \phi_{, x y\left(x^{\prime \prime}\right) y\left(x^{\prime}\right)} \mathrm{d} x^{\prime} \mathrm{d} x^{\prime \prime}} \\
& +\int_{G} \zeta_{; x x y\left(x^{\prime}\right)} \mathrm{d} x^{\prime} \frac{\partial}{\partial \phi_{, x x y\left(x^{\prime}\right)} \mathrm{d} x^{\prime}}
\end{aligned}
$$

Applying $X_{\text {modif }}^{(3)}$ to $F$, we get the equation:

$$
X_{\text {modif }}^{(3)} F=\zeta_{; t}-\int_{G} \xi_{y(x)} \mathrm{d} x\left(i \phi_{, x y(x) y(x)}+v \phi_{, x x y(x)}\right)-\int_{G} y(x)\left(i \zeta_{; x y(x) y(x)} \mathrm{d} x+\nu \zeta_{; x x y(x)} \mathrm{d} x\right)=0
$$

We insert the infinitesimals $\zeta_{; t}\left(c f\right.$. Equation (18)), $\zeta_{; x y(x) y(x)}$ and $\zeta_{; x x y(x)}(c f$. Appendix) and employ $F=0$ in order to eliminate $\phi_{, t}$.

In the extended Lie symmetry analysis, we deal with different types of derivatives. Inside the integral, the functional derivatives $\delta / \delta y(x)$ are present. It was argued that these derivatives correspond to partial derivatives $\partial / \partial y_{k}$ in the discrete case; $c f$. Section 2.1. Additionally, in (8), partial derivatives with respect to $x$ are present $\phi_{, x y(x) y(x)}$ and $\phi_{, x x y(x)}$, which do not have a correspondence in the discrete case. Furthermore, different types of mixed functional-partial derivatives are present in the final formula (23) In the further procedure, in order to obtain proper symmetry transformations, for the mixed derivatives, we had to assume that:

- $\phi_{, y(x)}$ is not independent of $\phi_{, x y(x)}$,

- $\phi_{, t y(x)}$ is not independent of $\phi_{, t x y(x)}$,

- $\phi_{, y(x) y(z)}$ is not independent of $\phi_{, x y(x) y(z)}$,

etc. Hence, in the final step, we integrate by parts in order to remove the $x$-derivatives from the functional derivatives of $\phi$. In order to do this, we have to eliminate the boundary integrals. One may choose between the following two options:

- The first option is to demand that $G \subseteq \mathbb{R}$ is bounded: $G=(a, b)$ with $-\infty<a<b<\infty$. If this holds true, one has to demand additionally that all appearing terms evaluated at $x=b$ are equal to the same terms evaluated at $x=a$. Then, all boundary integrals vanish. As this demands a huge number of restrictions, we do not choose $G$ to be bounded. Instead, we choose the second option. 
- The second option is to demand that $G \subseteq \mathbb{R}$ is not bounded. We restrict ourselves to the case $G=(-\infty,+\infty)$. If this holds true, one may impose the condition:

$$
U^{t}(x= \pm \infty)=0
$$

As Equation (5) states:

$$
\phi_{, y(x)}=i\left\langle U^{t}(x) e^{i\left(U^{t}, y\right)}\right\rangle
$$

we have:

$$
\phi,\left.y(x)\right|_{x= \pm \infty}=0
$$

Additionally, we impose that all functional derivatives of $\phi$ vanish for $x \rightarrow \pm \infty$, i.e.,

$$
\left.\phi_{, t y(x)}\right|_{x= \pm \infty}=0,\left.\quad \phi_{, x y(x)}\right|_{x= \pm \infty}=0,\left.\quad \phi_{, y(x) y(x)}\right|_{x= \pm \infty}=0,
$$

Hence, all appearing boundary integrals vanish. For example,

$$
\int_{G} \xi_{y(x)} \mathrm{d} x \phi_{, x y(x) y(x)}=-\int_{G} \frac{\partial \xi_{y(x)} \mathrm{d} x}{\partial x} \phi_{, y(x) y(x)}+\underbrace{\left[\xi_{y(x)} \phi_{, y(x) y(x)}\right]_{x=-\infty}^{x=+\infty}}_{=0}
$$

The resulting Equation (23) has the form:

$$
\begin{aligned}
0= & \mathcal{A}+\int_{G} \phi_{, y(z)} \mathcal{B} \mathrm{d} z+\int_{G} \int_{G} \phi_{, y(x) y(z)} \mathcal{C} \mathrm{d} z \mathrm{~d} x+\int_{G} \int_{G} \phi_{, y(x)} \phi_{, y(z)} \mathcal{D} \mathrm{d} z \mathrm{~d} x \\
& +\int_{G} \int_{G} \int_{G} \phi_{, y(x) y(a)} \phi_{, y(z)} \mathcal{E} \mathrm{d} a \mathrm{~d} z \mathrm{~d} x+\int_{G} \int_{G}\left(\phi_{, y(x)}\right)^{2} \phi_{, y(z)} \mathcal{F} \mathrm{d} z \mathrm{~d} x \\
& +\int_{G} \phi_{, t y(x)} \mathcal{G} \mathrm{d} x+\int_{G} \int_{G}\left(\phi_{, y(x)}\right)^{2} \phi_{, y(z) y(z)} \mathcal{H} \mathrm{d} z \mathrm{~d} x+\int_{G} \phi_{, y(x)} \phi_{, t y(x)} \mathcal{I} \mathrm{d} x \\
& +\int_{G} \phi_{, t y(x) y(x)} \mathcal{J} \mathrm{d} x+\int_{G} \int_{G} \phi_{, y(z) y(x) y(x)} \mathcal{K} \mathrm{d} z \mathrm{~d} x+\int_{G} \int_{G} \phi_{, y(x) y(x)} \phi_{, y(z) y(z)} \mathcal{L} \mathrm{d} z \mathrm{~d} x
\end{aligned}
$$

Since the infinitesimals $\xi_{y(z)} \mathrm{d} z, \xi_{x}, \xi_{t}, \eta_{\phi}$ do not depend on derivatives of $\phi$, all coefficients of all appearing derivatives of $\phi$ have to vanish:

$$
\mathcal{A}=\mathcal{B}=\mathcal{C}=\mathcal{D}=\mathcal{E}=\mathcal{F}=\mathcal{G}=\mathcal{H}=\mathcal{I}=\mathcal{J}=\mathcal{K}=\mathcal{L}=0
$$

This leads to the following system of linear FDEs for the infinitesimals $\xi_{y(z)} \mathrm{d} z, \xi_{x}, \xi_{t}, \eta_{\phi}$ :

- $\mathcal{A}=0$ reads:

$$
\frac{\partial \eta_{\phi}}{\partial t}-\int_{G} y(x)\left(i \frac{\partial^{3} \eta_{\phi}}{\partial x \partial(y(x) \mathrm{d} x)^{2}}+v \frac{\partial^{3} \eta_{\phi}}{\partial x^{2} \partial y(x) \mathrm{d} x}\right) \mathrm{d} x=0
$$


- $\mathcal{B}=0$ reads:

$$
\begin{aligned}
& 0=v \frac{\partial^{2}}{\partial z^{2}}\left(y(z) \frac{\partial \eta_{\phi}}{\partial \phi}\right)-v \frac{\partial^{2}}{\partial z^{2}}\left(y(z) \frac{\partial \xi_{t}}{\partial t}\right)-\frac{\partial \xi_{y(z)}}{\partial t}-v \frac{\partial^{2} \xi_{y(z)}}{\partial z^{2}} \\
& -2 i y(z) \frac{\partial^{3} \eta_{\phi}}{\partial z \partial y(z) \mathrm{d} z \partial \phi}+i v \int_{G} \frac{\partial^{2}}{\partial z^{2}}\left(y(x) y(z) \frac{\partial^{3} \xi_{t}}{\partial x \partial(y(x) \mathrm{d} x)^{2}}\right) \mathrm{d} x \\
& +i \int_{G} y(x) \frac{\partial^{3} \xi_{y(z)}}{\partial x \partial(y(x) \mathrm{d} x)^{2}} \mathrm{~d} x-i \frac{\partial}{\partial z}\left(y(z) \frac{\partial^{2} \xi_{z}}{\partial z \partial y(z) \mathrm{d} z}\right)+2 i \frac{\partial}{\partial z}\left(y(z) \frac{\partial^{2} \eta_{\phi}}{\partial y(z) \mathrm{d} z \partial \phi}\right) \\
& -i \int_{G} \frac{\partial}{\partial x}\left(y(x) \frac{\partial^{2} \xi_{y(z)}}{\partial(y(x) \mathrm{d} x)^{2}}\right) \mathrm{d} x+i \frac{\partial^{2}}{\partial z^{2}}\left(y(z) \frac{\partial \xi_{z}}{\partial y(z) \mathrm{d} z}\right) \\
& +v^{2} \int_{G} \frac{\partial^{2}}{\partial z^{2}}\left(y(x) y(z) \frac{\partial^{3} \xi_{t}}{\partial x^{2} \partial y(x) \mathrm{d} x}\right) \mathrm{d} x+\boldsymbol{v} \int_{G} y(x) \frac{\partial^{3} \xi_{y(z)}}{\partial x^{2} \partial y(x) \mathrm{d} x} \mathrm{~d} x \\
& -2 v \int_{G} \frac{\partial}{\partial x}\left(y(x) \frac{\partial^{2} \xi_{y(z)}}{\partial x \partial y(x) \mathrm{d} x}\right) \mathrm{d} x-v \frac{\partial}{\partial z}\left(y(z) \frac{\partial^{2} \xi_{z}}{\partial z^{2}}\right)-v \frac{\partial^{2}}{\partial z^{2}}\left(y(z) \frac{\partial \eta_{\phi}}{\partial \phi}\right) \\
& +v \int_{G} \frac{\partial^{2}}{\partial x^{2}}\left(y(x) \frac{\partial \xi_{y(z)}}{\partial y(x) \mathrm{d} x}\right) \mathrm{d} x+2 v \frac{\partial^{2}}{\partial z^{2}}\left(y(z) \frac{\partial \xi_{z}}{\partial z}\right)+y^{\prime}(z) \frac{\partial \xi_{z}}{\partial t}+y(z) \frac{\partial^{2} \xi_{z}}{\partial x \partial t} \\
& +i \frac{\partial^{2}}{\partial z^{2}}\left(y(z) \frac{\partial \xi_{z}}{\partial y(z) \mathrm{d} z}\right)-i \frac{\partial}{\partial z}\left(y(z) \frac{\partial^{2} \xi_{z}}{\partial z \partial y(z) \mathrm{d} z}\right)+i \int_{G} \frac{\partial^{2}}{\partial z \partial x}\left(y(z) y(x) \frac{\partial^{2} \xi_{z}}{\partial y(x) \mathrm{d} x \partial y(x) \mathrm{d} x}\right) \mathrm{d} x \\
& -i \int_{G} y(x) \frac{\partial}{\partial z}\left(y(z) \frac{\partial^{3} \xi_{z}}{\partial x(\partial y(x) \mathrm{d} x)^{2}}\right) \mathrm{d} x-v \int_{G} y^{\prime}(z) y(x) \frac{\partial^{3} \xi_{z}}{\partial x^{2} \partial y(x) \mathrm{d} x} \mathrm{~d} x \\
& -v \int_{G} y(z) y(x) \frac{\partial^{4} \xi_{z}}{\partial z \partial x^{2} \partial y(x) \mathrm{d} x} \mathrm{~d} x-v \int_{G} \frac{\partial^{3}}{\partial x^{2} \partial z}\left[y(z) y(x) \frac{\delta \xi_{z}}{\delta y(x)}\right] \mathrm{d} x \\
& +2 v \int_{G} \frac{\partial^{2}}{\partial x \partial z}\left[y(z) y(x) \frac{\partial \xi_{z}}{\partial x \partial y(x) \mathrm{d} x}\right] \mathrm{d} x
\end{aligned}
$$

- $\mathcal{C}=0$ reads:

$$
\begin{aligned}
0= & -i \frac{\partial}{\partial x}\left(y(x) \frac{\partial \eta_{\phi}}{\partial \phi}\right) \delta(x-z)+i \frac{\partial}{\partial x}\left(y(x) \frac{\partial \xi_{t}}{\partial t}\right) \delta(x-z)+i \frac{\partial \xi_{y(x)}}{\partial x} \delta(x-z) \\
& +\int_{G} \frac{\partial}{\partial z}\left(y(a) y(z) \frac{\partial^{3} \xi_{t}}{\partial a \partial(y(a) \mathrm{d} a)^{2}}\right) \delta(x-z) \mathrm{d} a+2 i y(x) \frac{\partial^{2} \xi_{y(z)}}{\partial x \partial y(x) \mathrm{d} x} \\
& -2 i \frac{\partial}{\partial x}\left(y(x) \frac{\partial \xi_{y(z)}}{\partial y(x) \mathrm{d} x}\right)+i \frac{\partial}{\partial x}\left(y(x) \frac{\partial \eta_{\phi}}{\partial \phi}\right) \delta(x-z)-i \frac{\partial}{\partial x}\left(y(x) \frac{\partial \xi_{x}}{\partial x}\right) \delta(x-z) \\
& -i v \int_{G} \frac{\partial}{\partial z}\left(y(a) y(z) \frac{\partial^{3} \xi_{t}}{\partial a^{2} \partial y(a) \mathrm{d} a}\right) \delta(x-z) \mathrm{d} a+v y(x) \frac{\partial^{3} \xi_{y(z)}}{\partial x^{2} \partial \phi}+v y(x) \frac{\partial^{2} \xi_{y(z)}}{\partial x^{2}} \\
& -v \frac{\partial}{\partial x}\left(y(x) \frac{\partial \xi_{y(z)}}{\partial x}\right)-v \frac{\partial}{\partial x}\left(y(x) \frac{\partial \xi_{y(z)}}{\partial x}\right)-2 i y(x) \frac{\partial}{\partial z}\left(y(z) \frac{\partial^{2} \xi_{z}}{\partial x \partial y(x) \mathrm{d} x}\right) \\
& +2 i \frac{\partial^{2}}{\partial x \partial z}\left(y(x) y(z) \frac{\partial \xi_{z}}{\partial y(x) \mathrm{d} x}\right)
\end{aligned}
$$


- $\mathcal{D}=0$ reads:

$$
\begin{aligned}
0= & -v^{2} \frac{\partial^{4}}{\partial z^{2} \partial x^{2}}\left(y(x) y(z) \frac{\partial \xi_{t}}{\partial \phi}\right)-v \frac{\partial^{2}}{\partial x^{2}}\left(y(x) \frac{\partial \xi_{y(z)}}{\partial \phi}\right)+2 i v \frac{\partial^{2}}{\partial z^{2}}\left(y(x) y(z) \frac{\partial^{3} \xi_{t}}{\partial x \partial y(x) \mathrm{d} x \partial \phi}\right) \\
& +2 i y(x) \frac{\partial^{3} \xi_{y(z)}}{\partial x \partial y(x) \mathrm{d} x \partial \phi}-i \frac{\partial}{\partial x}\left(y(x) \frac{\partial^{2} \xi_{x}}{\partial x \partial \phi}\right) \delta(x-z)-2 i v \frac{\partial^{3}}{\partial z^{2} \partial x}\left(y(x) y(z) \frac{\partial^{2} \xi_{t}}{\partial y(x) \mathrm{d} x \partial \phi}\right) \\
& -2 i \frac{\partial}{\partial x}\left(y(x) \frac{\partial^{2} \xi_{y}(z)}{\partial y(x) \mathrm{d} x \partial \phi}\right) \delta(x-z)-2 i \frac{\partial}{\partial x}\left(y(x) \frac{\partial^{2} \xi_{y(z)}}{\partial y(x) \mathrm{d} x \partial \phi}\right) \\
& +2 i \frac{\partial}{\partial x}\left(y(x) \frac{\partial^{2} \eta_{\phi}}{\partial \phi^{2}}\right) \delta(x-z)+i \frac{\partial^{2}}{\partial x^{2}}\left(y(x) \frac{\partial \xi_{x}}{\partial \phi}\right) \delta(x-z)+i \frac{\partial^{2}}{\partial x^{2}}\left(y(x) \frac{\partial \xi_{x}}{\partial \phi}\right) \delta(x-z) \\
& -v \frac{\partial}{\partial x}\left(y(x) \frac{\partial^{2} \xi_{y(z)}}{\partial x \partial \phi}\right)-2 v \frac{\partial}{\partial x}\left(y(x) \frac{\partial^{2} \xi_{y(z)}}{\partial x \partial \phi}\right)-v \frac{\partial}{\partial x}\left(y(x) \frac{\partial^{2} \xi_{y}(z)}{\partial x \partial \phi}\right) \\
& +2 v \frac{\partial^{2}}{\partial x^{2}}\left(y(x) \frac{\partial \xi_{y(z)}}{\partial \phi}\right)+v^{2} \frac{\partial^{4}}{\partial z^{2} \partial x^{2}}\left(y(x) y(z) \frac{\partial \xi_{t}}{\partial \phi}\right)+v \frac{\partial^{2}}{\partial x^{2}}\left(y(x) \frac{\partial \xi_{y(z)}}{\partial \phi}\right) \\
& +v \frac{\partial^{2}}{\partial x^{2}}\left(y(x) \frac{\partial \xi_{y(z)}}{\partial \phi}\right)-2 v^{2} \frac{\partial^{3}}{\partial z^{2} \partial x}\left(y(x) y(z) \frac{\partial^{2} \xi_{t}}{\partial x \partial \phi}\right)+v^{2} \frac{\partial^{2}}{\partial z^{2}}\left(y(x) y(z) \frac{\partial^{3} \xi_{t}}{\partial x^{2} \partial \phi}\right) \\
& +i \frac{\partial^{2}}{\partial x^{2}}\left(y(x) \frac{\partial \xi_{x}}{\partial \phi} \delta(x-z)\right)+i \frac{\partial^{2}}{\partial x \partial z}\left(y(x) \frac{\partial \xi_{x}}{\partial \phi} \delta(x-z)\right)-i \frac{\partial}{\partial x}\left(y(x) \frac{\partial^{2} \xi_{x}}{\partial \phi \partial x} \delta(x-z)\right) \\
& -i \frac{\partial}{\partial z}\left(y(x) y(z) \frac{\partial^{3} \xi_{z}}{\partial x \partial \phi \delta y(x)}\right)+2 i \frac{\partial^{2}}{\partial z \partial x}\left(y(x) y(z) \frac{\partial^{2} \xi_{z}}{\partial \phi \delta y(x)}\right)
\end{aligned}
$$

- $\mathcal{E}=0$ reads:

$$
\begin{aligned}
0= & 2 i v \frac{\partial^{3}}{\partial z^{2} \partial x}\left(y(x) y(z) \frac{\partial \xi_{t}}{\partial \phi}\right) \delta(x-a)+i \frac{\partial}{\partial x}\left(y(x) \frac{\partial \xi_{y(z)}}{\partial \phi}\right) \delta(x-a) \\
& +2 \frac{\partial}{\partial x}\left(y(x) y(z) \frac{\partial^{3} \xi_{t}}{\partial z \partial y(z) \mathrm{d} z \partial \phi}\right) \delta(x-a)+2 i y(z) \frac{\partial^{2} \xi_{y(x)}}{\partial z \partial \phi} \delta(a-z)+i y(x) \frac{\partial^{2} \xi_{y(z)}}{\partial x \partial \phi} \delta(x-a) \\
& -2 \frac{\partial^{2}}{\partial z \partial x}\left(y(x) y(z) \frac{\partial^{2} \xi_{t}}{\partial y(z) \mathrm{d} z \partial \phi}\right) \delta(x-a)-2 i \frac{\partial}{\partial z}\left(y(z) \frac{\partial \xi_{y(x)}}{\partial \phi}\right) \delta(a-z) \\
& -i \frac{\partial}{\partial x}\left(y(x) \frac{\partial \xi_{y(z)}}{\partial \phi}\right) \delta(x-a)-2 i \frac{\partial}{\partial z}\left(y(z) \frac{\partial \xi_{y}(x)}{\partial \phi}\right) \delta(a-z) \\
& -i v \frac{\partial^{3}}{\partial z^{2} \partial x}\left(y(x) y(z) \frac{\partial \xi_{t}}{\partial \phi}\right) \delta(x-a)-i \frac{\partial}{\partial x}\left(y(x) \frac{\partial \xi_{y(z)}}{\partial \phi}\right) \delta(x-a) \\
& -i v \frac{\partial^{3}}{\partial z^{2} \partial x}\left(y(x) y(z) \frac{\partial \xi_{t}}{\partial \phi}\right) \delta(x-a)+i v \frac{\partial^{2}}{\partial z^{2}}\left(y(x) y(z) \frac{\partial^{2} \xi_{t}}{\partial x \partial \phi}\right) \delta(x-a) \\
& +2 i v \frac{\partial^{2}}{\partial z \partial x}\left(y(x) y(z) \frac{\partial^{2} \xi_{t}}{\partial z \partial \phi}\right) \delta(x-a)-2 i v \frac{\partial}{\partial x}\left(y(x) y(z) \frac{\partial^{3} \xi_{t}}{\partial z^{2} \partial \phi}\right) \delta(x-a) \\
& +i y^{\prime}(z) y^{\prime}(x) \delta(a-z) \frac{\partial \xi_{z}}{\partial \phi}
\end{aligned}
$$

- $\mathcal{F}=0$ reads:

$$
\begin{aligned}
0= & i y(x) \frac{\partial^{3} \xi_{y(z)}}{\partial x \partial \phi^{2}}-2 i v \frac{\partial^{3}}{\partial z^{2} \partial x}\left(y(x) y(z) \frac{\partial^{2} \xi_{t}}{\partial \phi^{2}}\right)-i \frac{\partial}{\partial x}\left(y(x) \frac{\partial^{2} \xi_{y(z)}}{\partial \phi^{2}}\right) \\
& -2 i \frac{\partial}{\partial x}\left(y(x) \frac{\partial^{2} \xi_{y(z)}}{\partial \phi^{2}}\right)+i v \frac{\partial^{2}}{\partial z^{2}}\left(y(x) y(z) \frac{\partial^{3} \xi_{t}}{\partial x \partial \phi^{2}}\right)
\end{aligned}
$$


- $\mathcal{G}=0$ reads:

$$
\begin{aligned}
0= & 2 i y(x) \frac{\partial^{2} \xi_{t}}{\partial x \partial y(x) \mathrm{d} x}-2 i \frac{\partial}{\partial x}\left(y(x) \frac{\partial \xi_{t}}{\partial y(x) \mathrm{d} x}\right)-v \frac{\partial}{\partial x}\left(y(x) \frac{\partial \xi_{t}}{\partial x}\right)+v y(x) \frac{\partial^{2} \xi_{t}}{\partial x^{2}} \\
& -v \frac{\partial}{\partial x}\left(y(x) \frac{\partial \xi_{t}}{\partial x}\right)
\end{aligned}
$$

- $\mathcal{H}=0$ reads

$$
0=-2 \frac{\partial^{2}}{\partial z \partial x}\left(y(x) y(z) \frac{\partial^{2} \xi_{t}}{\partial \phi^{2}}\right)+\frac{\partial}{\partial z}\left(y(x) y(z) \frac{\partial^{3} \xi_{t}}{\partial x \partial \phi^{2}}\right)
$$

- $\mathcal{I}=0$ reads:

$$
0=-2 i \frac{\partial}{\partial x}\left(y(x) \frac{\partial \xi_{t}}{\partial \phi}\right)-2 i \frac{\partial}{\partial x}\left(y(x) \frac{\partial \xi_{t}}{\partial \phi}\right)+2 i y(x) \frac{\partial^{2} \xi_{t}}{\partial x \partial \phi}
$$

- $\mathcal{J}=0$ reads:

$$
0=i y(x) \frac{\partial \xi_{t}}{\partial x}
$$

- $\mathcal{K}=0$ reads:

$$
0=i y(x) \frac{\partial \xi_{y(z)}}{\partial x}
$$

- $\mathcal{L}=0$ reads

$$
0=\frac{\partial^{2}}{\partial z \partial x}\left(y(x) y(z) \frac{\partial \xi_{t}}{\partial \phi}\right)-\frac{\partial^{2}}{\partial z \partial x}\left(y(x) y(z) \frac{\partial \xi_{t}}{\partial \phi}\right)-\frac{\partial}{\partial z}\left(y(x) y(z) \frac{\partial^{2} \xi_{t}}{\partial x \partial \phi}\right)
$$

3.2.2. Solution of the Determining System of Equations for the Infinitesimals

- First of all, consider Equation (35). Since this equation has to hold for all choices of $y \in L^{2}(G, \mathbb{R})$, the coefficient of $y$ has to vanish, and we get:

$$
\frac{\partial \xi_{y(z)}}{\partial x}=0
$$

- Now, consider Equation (34). Similarly, we get:

$$
\frac{\partial \xi_{t}}{\partial x}=0
$$

- Then, consider Equation (33). Similarly, we get:

$$
\frac{\partial \xi_{t}}{\partial \phi}=0
$$

- Due to Equation (39), Equations (32) and (36) are fulfilled identically.

- Next, we consider Equation (31). We apply the product rule and make use of Equation (38). We get:

$$
\frac{\partial \xi_{t}}{\partial y(x) \mathrm{d} x}=0
$$

Considering Equations (38) and (39), we have $\xi_{t}=\xi_{t}(t)$. 
- If we apply Equations (39) and (37) to Equation (29), we obtain:

$$
-4 i \frac{\partial}{\partial z}\left(y(z) \frac{\partial \xi_{y(x)}}{\partial \phi}\right) \delta(a-z)-i \frac{\partial}{\partial x}\left(y(x) \frac{\partial \xi_{y(z)}}{\partial \phi}\right) \delta(x-a)+i y^{\prime}(z) y^{\prime}(x) \delta(a-z) \frac{\partial \xi_{z}}{\partial \phi}=0
$$

Considering the case $a \neq z$, and taking into account that $y(x)$ is an arbitrary function, we get:

$$
\frac{\partial \xi_{y(z)}}{\partial \phi}=0
$$

With the above relation, Equation (41) for $a \neq x$ leads to:

$$
\frac{\partial \xi_{z}}{\partial \phi}=0
$$

which holds for each $z \in G$. If we substitute this result back into Equation (41) and assume $x=z$, we find again Formula (42), which has to hold for each $z \in G$. As $y(z)$ is an arbitrary function, Equation (41) is fulfilled for $x \neq z$, as well.

- By virtue of Equations (39) and (42), Equation (30) is fulfilled identically.

- Now, we take a look at the remaining four Equations (25)-(28). We start with Equation (28). Considering Equations (39), (42) and (43), Equation (28) reads:

$$
2 i \frac{\partial}{\partial x}\left(y(x) \frac{\partial^{2} \eta_{\phi}}{\partial \phi^{2}}\right) \delta(x-z)=0
$$

hence:

$$
\frac{\partial^{2} \eta_{\phi}}{\partial \phi^{2}}=0
$$

Equation (44) means that there are functionals $f, g$, such that:

$$
\eta_{\phi}=f\left(\left[y\left(x^{\prime}\right)\right], t\right) \phi+g\left(\left[y\left(x^{\prime}\right)\right], t\right)
$$

For $f$, we choose the ansatz:

$$
f\left(\left[y\left(x^{\prime}\right)\right], t\right)=\int_{G} f_{1}\left(x^{\prime}, t\right) y\left(x^{\prime}\right) \mathrm{d} x^{\prime}+f_{2}(t)
$$

- The next equation we solve is Equation (27). If we use Equations (40) and (37) and apply the product rule, Equation (27) reads:

$$
\begin{aligned}
0= & i \frac{\partial}{\partial x}\left(y(x) \frac{\partial \xi_{t}}{\partial t}\right) \delta(x-z)+i \frac{\partial \xi_{y(x)}}{\partial x} \delta(x-z) \\
& -i \frac{\partial}{\partial x}\left(y(x) \frac{\partial \xi_{x}}{\partial x}\right) \delta(x-z)-2 i y^{\prime}(x) \frac{\partial \xi_{y(z)}}{\partial y(x) \mathrm{d} x}+2 i y^{\prime}(z) y^{\prime}(x) \frac{\partial \xi_{z}}{\partial y(x) \mathrm{d} x}
\end{aligned}
$$

Considering the case $x \neq z$, we get:

$$
y^{\prime}(z) \frac{\partial \xi_{z}}{\partial y(x) \mathrm{d} x}-\frac{\partial \xi_{y(z)}}{\partial y(x) \mathrm{d} x}=0
$$


Although Equation (48) allows a broader range of solutions, we will restrict our considerations to the case:

$$
\frac{\partial \xi_{z}}{\partial y(x) \mathrm{d} x}=0, \quad \frac{\partial \xi_{y(z)}}{\partial y(x) \mathrm{d} x}=0
$$

and use the following ansatz's for $\xi_{y(z)} \mathrm{d} z$ and $\xi_{z}$ :

$$
\xi_{y(z)} \mathrm{d} z=c(z, t) \mathrm{d} z+c_{0}(z, t) y(z) \mathrm{d} z, \quad \xi_{z}=c_{1}(z, t)+c_{2}(z, t) y(z)
$$

Next, we want to consider Equation (47) without the restriction $x \neq z$; hence, we integrate Equation (47) with respect to $z \in G$. This leads to:

$\frac{\partial}{\partial x}\left(y(x) \frac{\partial \xi_{t}}{\partial t}\right)+\frac{\partial \xi_{y(x)}}{\partial x}-\frac{\partial}{\partial x}\left(y(x) \frac{\partial \xi_{x}}{\partial x}\right)-2 \int_{G} y^{\prime}(x) \frac{\partial \xi_{y(z)}}{\partial y(x) \mathrm{d} x} \mathrm{~d} z+2 y^{\prime}(x) \int_{G} y^{\prime}(z) \frac{\partial \xi_{z}}{\partial y(x) \mathrm{d} x} \mathrm{~d} z=0$.

Now, we put in Ansatz (50), make use of $\xi_{t}=\xi_{t}(t)$ and take into consideration that this equation has to hold for all choices of $y \in L^{2}(G, \mathbb{R})$; hence, the coefficients of one, $y, y^{\prime}, y^{\prime \prime}, y y^{\prime}, y^{\prime} y^{\prime}$ have to vanish:

$$
\begin{aligned}
\frac{\partial c(x, t)}{\partial x} & =0 \Longrightarrow c=c(t), \\
\frac{\partial c_{0}(x, t)}{\partial x}-\frac{\partial^{2} c_{1}}{\partial x^{2}} & =0 \\
\xi_{t}^{\prime}(t)-\frac{\partial c_{1}}{\partial x}-c_{0}(x, t) & =0, \\
c_{2}(x, t) & =0
\end{aligned}
$$

After differentiating Equation (53) with respect to $x$, we find:

$$
\frac{\partial c_{0}}{\partial x}+\frac{\partial^{2} c_{1}}{\partial x^{2}}=0
$$

which, together with Equation (52), gives:

$$
\frac{\partial^{2} c_{1}}{\partial x^{2}}=0, \quad c_{0}=c_{0}(t)
$$

Considering this and Equations (51) and (53), Ansatz (50) reads:

$$
\begin{aligned}
\xi_{z} & =c_{3}(t) z+c_{4}(t) . \\
\xi_{y(z)} \mathrm{d} z & =c(t) \mathrm{d} z+\left(\xi_{t}^{\prime}(t)-c_{3}(t)\right) y(z) \mathrm{d} z,
\end{aligned}
$$

- Now, we are ready to deal with Equation (26). If we use Equations (56), (57), (40), (46) and (55), Equation (26) reads:

$$
\begin{aligned}
0= & -\boldsymbol{v} y^{\prime \prime}(z) \xi_{t}^{\prime}(t)-c^{\prime}(t)-\left(\xi_{t}^{\prime \prime}(t)-\frac{\partial^{2} \xi_{x}}{\partial t \partial x}(t)\right) y(z)-v\left(\xi_{t}^{\prime}(t)-\frac{\partial \xi_{x}}{\partial x}(t)\right) y^{\prime \prime}(z) \\
& -2 i y(z) \frac{\partial f_{1}(z, t)}{\partial z}+2 i \frac{\partial}{\partial z}\left(y(z) f_{1}(z, t)\right)+v \int_{G} y(x)\left(\xi_{t}^{\prime}(t)-\frac{\partial \xi_{z}}{\partial z}(t)\right) \frac{\partial^{2} \delta(x-z)}{\partial x^{2}} \mathrm{~d} x \\
& -2 v \int_{G} \frac{\partial}{\partial x}\left(y(x)\left(\xi_{t}^{\prime}(t)-\frac{\partial \xi_{x}}{\partial x}(t)\right) \frac{\partial \delta(x-z)}{\partial x}\right) \mathrm{d} x-v \frac{\partial}{\partial z}\left(y(z) \frac{\partial^{2} \xi_{z}}{\partial z^{2}}\right) \\
& +v \int_{G} \frac{\partial^{2}}{\partial x^{2}}\left(y(x)\left(\xi_{t}^{\prime}(t)-\frac{\partial \xi_{x}}{\partial x}(t)\right) \delta(x-z)\right) \mathrm{d} x+2 v \frac{\partial^{2}}{\partial z^{2}}\left(y(z) \frac{\partial \xi_{z}}{\partial z}(t)\right)+y^{\prime}(z) \frac{\partial \xi_{z}}{\partial t} \\
& +y(z) \frac{\partial^{2} \xi_{z}}{\partial t \partial x}
\end{aligned}
$$


In this equation, the last two integrals involving the Dirac delta distribution vanish if we assume that:

$$
y(x), y^{\prime}(x) \longrightarrow 0 \text { for } x \rightarrow \pm \infty
$$

As Equation (58) has to hold for all choices of $y \in L^{2}(G, \mathbb{R})$, the coefficients of one, $y, y^{\prime}$ and $y^{\prime \prime}$ have to vanish. We evaluate the coefficient of $y^{\prime \prime}$ in $x=z$ and get:

$$
\begin{aligned}
-c^{\prime}(t) & =0 \Longrightarrow c(t)=\text { const. }=: c \in \mathbb{R}, \\
-\xi_{t}^{\prime \prime}(t)+2 \frac{\partial^{2} \xi_{z}}{\partial t \partial z}(t) & =0, \\
2 i f_{1}(z, t)+\frac{\partial \xi_{z}}{\partial t} & =0, \\
2 \vee \frac{\partial \xi_{z}}{\partial z}(t)-v \xi_{t}^{\prime}(t) & =0
\end{aligned}
$$

From the above system, we first use the relation (62), (45), take into account (55) and substitute into Equation (25) to get:

$$
\frac{1}{2} i \frac{\partial^{2} \xi_{z}}{\partial t^{2}} \int y(x) \mathrm{d} x \phi+\frac{\partial g}{\partial t}-\int_{G} y(x)\left(i \frac{\partial^{3} g}{\partial x \partial(y(x) \mathrm{d} x)^{2}}+\boldsymbol{v} \frac{\partial^{3} g}{\partial x^{2} \partial y(x) \mathrm{d} x}\right) \mathrm{d} x=0
$$

This equation has to hold for every $\phi$; hence, the coefficient of $\phi$ has to vanish. This, together with (55) furnishes:

$$
\frac{\partial^{2} \xi_{z}}{\partial t^{2}}=0
$$

Hence, Equation (63) reads:

$$
\frac{\partial g}{\partial t}-\int_{G} y(x)\left(i \frac{\partial^{3} g}{\partial x \partial(y(x) \mathrm{d} x)^{2}}+v \frac{\partial^{3} g}{\partial x^{2} \partial y(x) \mathrm{d} x}\right) \mathrm{d} x=0
$$

i.e., $g$ has to fulfill the viscous Hopf-Burgers FDE. This is expected by the classical Lie symmetry analysis, as the considered differential equation is linear; we see that this result is furnished by the extended Lie symmetry analysis, as well. With (64) the system (60-62) has the solution:

$$
\begin{aligned}
\xi_{t} & =2 a_{1} t+a_{2}+a_{4} t^{2}, \\
\xi_{x} & =a_{1} x+a_{3}+t x a_{4}+a_{5} t, \\
f_{1} & =\frac{1}{2} i x a_{1}+\frac{1}{2} i a_{3}+a_{6}
\end{aligned}
$$

where $a_{1}, a_{2}, a_{3}, a_{4}, a_{5}, a_{6} \in \mathbb{R}$

We insert Equations (67), (66) and (59) into Equation (57) to get:

$$
\xi_{y(z)} \mathrm{d} z=\left(a_{1}+t a_{4}\right) y(z) \mathrm{d} z+a_{7} \mathrm{~d} z
$$

with $\quad a_{7} \in \mathbb{R}$. 
Theorem 9 (Local transformations of the viscous Hopf-Burgers FDE). Finally, the infinitesimals of the viscous Hopf-Burgers FDE are given by:

$$
\begin{aligned}
\xi_{t} & =2 a_{1} t+a_{2}+a_{4} t^{2}, \\
\xi_{x} & =a_{1} x+a_{3}+t x a_{4}+a_{5} t \\
\xi_{y(z)} \mathrm{d} z & =\left(a_{1}+t a_{4}\right) y(z) \mathrm{d} z+a_{6} \mathrm{~d} z, \\
\eta_{\phi} & =\left(\frac{1}{2} i \int\left(a_{4} x+a_{5}\right) y(x) \mathrm{d} x+a_{7}\right) \phi+g\left(\left[y\left(x^{\prime}\right)\right], t\right)
\end{aligned}
$$

where $a_{1}, a_{2}, a_{3}, a_{4}, a_{5}, a_{6}, a_{7} \in \mathbb{R}$ are arbitrary constants and $g$ is an arbitrary functional, which has to fulfill the viscous Hopf-Burgers FDE.

The associated generators read:

$$
\begin{aligned}
X_{1} & =x \frac{\partial}{\partial x}+2 t \frac{\partial}{\partial t}+\int_{G} y(x) \mathrm{d} x \frac{\partial}{\partial y(x) \mathrm{d} x}, \\
X_{2} & =\frac{\partial}{\partial t} \\
X_{3} & =\frac{\partial}{\partial x} \\
X_{4} & =2 t x \frac{\partial}{\partial x}+2 t^{2} \frac{\partial}{\partial x}+2 t \int_{G} y(x) \mathrm{d} x \frac{\partial}{\partial y(x) \mathrm{d} x}+i \int x y(x) \mathrm{d} x \phi \frac{\partial}{\partial \phi} \\
X_{5} & =2 t \frac{\partial}{\partial x}+i \int y(x) \mathrm{d} x \phi \frac{\partial}{\partial \phi} \\
X_{6} & =\int_{G} \mathrm{~d} x \frac{\partial}{\partial y(x) \mathrm{d} x} \\
X_{7} & =\phi \frac{\partial}{\partial \phi}, \\
X_{g} & =g\left(\left[y\left(x^{\prime}\right)\right], t\right) \frac{\partial}{\partial \phi}
\end{aligned}
$$

\subsection{Symmetry Breaking Restrictions}

The Lie symmetry analysis furnishes symmetries of the viscous Hopf-Burgers FDE without respecting physical restrictions. If we incorporate such physical restrictions, we lose some of the calculated symmetries, which the viscous Hopf-Burgers FDE exhibits considered as a mathematical equation detached from any physical conditions. The loss of symmetries by incorporating physical restrictions is called symmetry breaking. This section is devoted to restrictions on $\phi$ breaking some of the calculated symmetries $X_{1}, \ldots, X_{g}$.

In [4], Hopf states conditions which have to be fulfilled by Hopf functionals:

$$
\phi([\boldsymbol{y}(\boldsymbol{x})], t)=\left\langle e^{i\left(\boldsymbol{U}^{t}, \boldsymbol{y}\right)}\right\rangle=\int_{L^{2}\left(G, \mathbb{R}^{3}\right)} e^{i(\boldsymbol{v}, \boldsymbol{y})} f^{t}([\boldsymbol{v}(\boldsymbol{x})]) \mathrm{d}[\boldsymbol{v}(\boldsymbol{x})]
$$

cf. Equation (3). These conditions may be derived from conditions that are imposed on the associated probability density functional $f^{t}$. The definition of a probability density functional requires $f^{t}$ to fulfill the following two conditions: 
Definition 10 (Probability density functional). $f^{t}$ is called a probability density functional if and only if:

1. $f^{t}$ is real-valued and non-negative, i.e.,

$$
f^{t}([\boldsymbol{v}(\boldsymbol{x})]) \in \mathbb{R}_{0}^{+}
$$

2. The integral of $f^{t}$ over the whole domain of integration equals one, i.e.,

$$
\int_{L^{2}\left(G, \mathbb{R}^{3}\right)} f^{t}([\boldsymbol{v}(\boldsymbol{x})]) \mathrm{d}[\boldsymbol{v}(\boldsymbol{x})]=1
$$

As in this paper, we restrict ourselves to the one-dimensional case and make use of the viscous Burgers equation instead of the incompressible Navier-Stokes equations; solutions of the viscous Hopf-Burgers FDE do not have to fulfill any conditions related to incompressibility. There remain three conditions that a solution $\phi$ of the viscous Hopf-Burgers FDE has to fulfill. We define:

Definition 11 (Physically-relevant solution of the viscous Hopf-Burgers FDE). Let $\phi$ be a solution of the viscous Hopf-Burgers FDE. $\phi$ is a physically-relevant solution of the viscous Hopf-Burgers FDE if and only if:

1. $\phi^{*}([y(x)], t)=\phi([-y(x)], t)$ where $\phi^{*}$ denotes the complex conjugate of $\phi$,

2. $\phi(0, t)=1$,

3. $|\phi([y(x)], t)| \leq 1$.

These three conditions are implied by Restriction (69) and Equation (70).

In Subsection 3.2.2, we showed that the extended Lie symmetry analysis furnishes eight generators $X_{1}, X_{2}, X_{3}, X_{4}, X_{5}, X_{6}, X_{7} X_{g}$, as the local transformations depend on seven parameters $a_{1}, a_{2}, a_{3}, a_{4}, a_{5}, a_{6}, a_{7}$ and on a functional $g$. Especially, the generators $X_{7}$ and $X_{g}$ associated with symmetries of $\phi$ are independent. As the conditions given by Definition 11 do not influence any symmetries corresponding to transformations of the independent variables $\left(\left[y\left(x^{\prime}\right)\right], x, t\right)$, the generators associated with transformations of the independent variables are not changed if we are looking for physically-relevant solutions: We have:

$$
X_{i}^{\text {phys }}=X_{i}, \quad i \in\{1,2,3,4,5\}
$$

Thus, it suffices to have a look at $X_{7}$ and $X_{g}$. If $\phi$ shall be a physically-relevant solution, $X_{7}$ and $X_{g}$ are not independent. In order to see this, decompose $g$ in:

$$
X_{g}=g\left(\left[y\left(x^{\prime}\right)\right], t\right) \frac{\partial}{\partial \phi}
$$

into a constant part $g_{1} \in \mathbb{C}$ and a non-constant part $g_{2}=g_{2}\left(\left[y\left(x^{\prime}\right)\right], t\right)$, i.e.,

$$
g\left(\left[y\left(x^{\prime}\right)\right], t\right)=g_{1}+g_{2}\left(\left[y\left(x^{\prime}\right)\right], t\right)
$$


Here, $g_{2}$ is a solution of the viscous Hopf-Burgers FDE; however, it is not a characteristic functional, i.e., the conditions given by Definition 11 must be fulfilled for the transformed functional $\bar{\phi}$, but not for $g_{2}$ separately. We get the decomposition $X_{g}=X_{g_{1}}+X_{g_{2}}$ with:

$$
\begin{aligned}
X_{g_{1}} & :=g_{1} \frac{\partial}{\partial \phi}, \quad g_{1} \in \mathbb{C}, \\
X_{g_{2}} & :=g_{2}\left(\left[y\left(x^{\prime}\right)\right], t\right) \frac{\partial}{\partial \phi}
\end{aligned}
$$

We replace $X_{7}$ and $X_{g}$ by the two generators:

$$
\begin{aligned}
X_{7}+X_{g_{1}} & =\left(\phi+g_{1}\right) \frac{\partial}{\partial \phi}, \quad f, g_{1} \in \mathbb{C}, \\
X_{g_{2}} & =g_{2}\left(\left[y\left(x^{\prime}\right)\right], t\right) \frac{\partial}{\partial \phi}
\end{aligned}
$$

and calculate the associated global transformations by solving the Lie initial value problems:

$$
\begin{array}{ll}
\frac{\partial \bar{\phi}}{\partial \epsilon}=\bar{\phi}+g_{1}, & \frac{\partial \bar{\phi}}{\partial \epsilon}=g_{2}\left(\left[y\left(x^{\prime}\right)\right], t\right), \\
\bar{\phi}(\epsilon=0)=\phi, & \bar{\phi}(\epsilon=0)=\phi
\end{array}
$$

The solutions are given by:

$$
\begin{aligned}
& \bar{\phi}([y(x)], t)=\phi([y(x)], t) e^{\epsilon}+\left(e^{\epsilon}-1\right) g_{1}, \quad \in \in \mathbb{R}, \quad g_{1} \in \mathbb{C} \\
& \bar{\phi}([y(x)], t)=\phi([y(x)], t)+g_{2}([y(x)], t) \epsilon, \quad \in \in \mathbb{R}
\end{aligned}
$$

In the following, we investigate the consequences of the conditions given by Definition 11 for $\in \in \mathbb{R}$ and $g_{1} \in \mathbb{C}$ if $\bar{\phi}$ is given by Equation (71).

1. As $\phi$ has to fulfill $\phi^{*}([y(x)], t)=\phi([-y(x)], t)$, we have:

$$
g_{1} \in \mathbb{R}
$$

2. As $\phi$ has to fulfill $\phi^{*}([y(x)], t)=\phi([-y(x)], t)$ and $\phi(0, t)=1$ and since $g_{1} \in \mathbb{R}$, we get:

$$
g_{1}=-1
$$

This shows that $X_{7}$ and $X_{g}$ are not independent.

3. As $\phi$ has to fulfill $|\phi([y(x)], t)| \leq 1$, we have:

$$
\epsilon \in(-\infty, 0]
$$

and the generator $X_{7}+X_{g_{1}}$ associated with the physically-relevant symmetry: reads

$$
X_{7}^{\text {phys }}:=\left(X_{7}+X_{g_{1}}\right)^{\text {phys }}=(\phi-1) \frac{\partial}{\partial \phi}
$$

Next, we investigate the consequences of the conditions given by Definition 11 for $\epsilon \in \mathbb{R}$ and $g_{2}=$ $g_{2}([y(x)], t)$ if $\bar{\phi}$ is given by Equation (72). 
1. As $\phi$ has to fulfill condition $\phi^{*}([y(x)], t)=\phi([-y(x)], t)$, we have:

$$
g_{2}^{*}([y(x)], t)=g_{2}([-y(x)], t)
$$

2. As $\phi$ has to fulfill $\phi^{*}([y(x)], t)=\phi([-y(x)], t)$ and $\phi(0, t)=1$, using Equation (73), we get:

$$
g_{2}(0, t)=0
$$

3. As $\phi$ has to fulfill condition $|\phi([y(x)], t)| \leq 1$, we have:

$$
\operatorname{Re}(\phi) \operatorname{Re}\left(g_{2} \epsilon\right)+\operatorname{Im}(\phi) \operatorname{Im}\left(g_{2} \epsilon\right) \leq 0
$$

Altogether, the generator $X_{g_{2}}$ associated with the physically-relevant symmetry reads:

$$
X_{g}^{\text {phys }}:=X_{g_{2}}^{\text {phys }}=g_{2}\left(\left[y\left(x^{\prime}\right)\right], t\right) \frac{\partial}{\partial \phi}
$$

where $g_{2}$ fulfills Conditions (73)-(75).

At the end of this section, we want to compare the calculated physically-relevant symmetries with the symmetries of the viscous Burgers equation; $c f$. [13]. Results are summarised in the Table 1.

Table 1. Comparison between the symmetries of the viscous Hopf-Burgers functional differential equation (FDE) and the viscous Burgers equation.

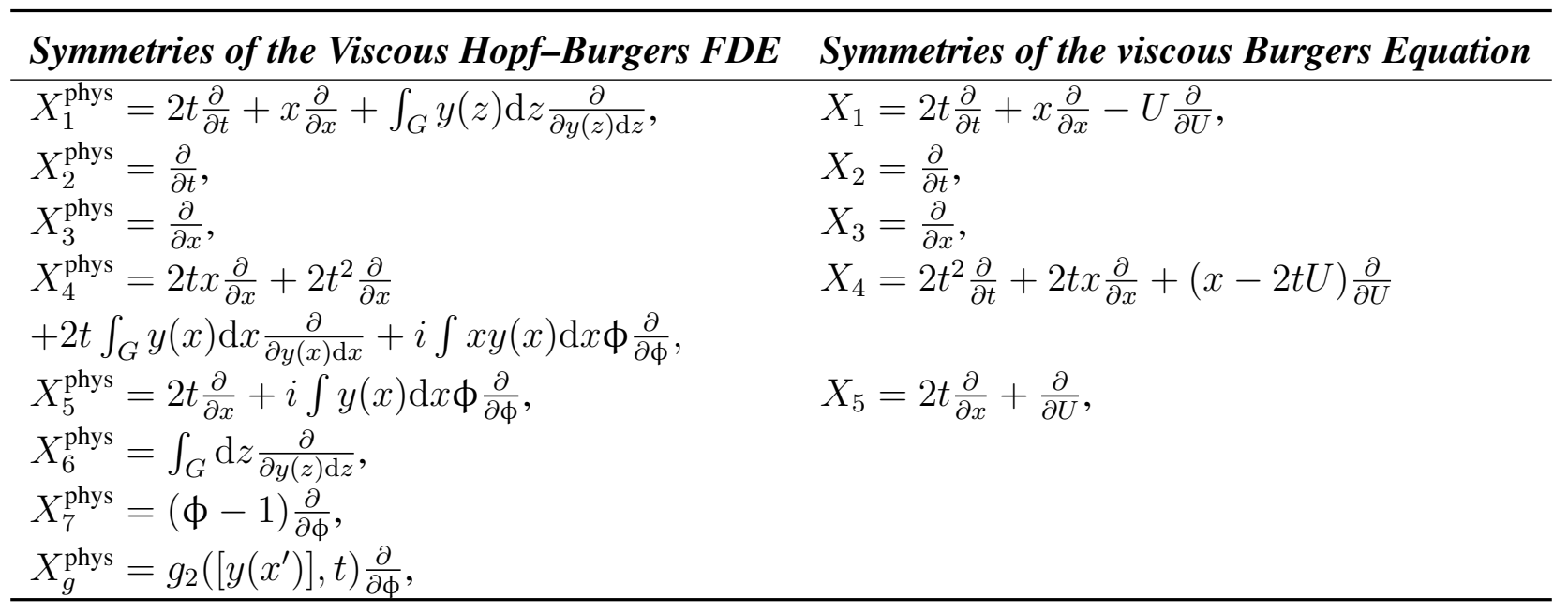

In Table $1, g_{2}$ is a solution of the viscous Hopf-Burgers FDE satisfying the three conditions (73)-(75). For $X_{7}^{\text {phys }}$, the group parameter $\epsilon$ is restricted to be non-positive, i.e., $\epsilon \in(-\infty, 0]$. For all of the other generators, we have $\in \in \mathbb{R}$.

We see that we rediscover the analogous forms of the symmetries of the viscous Burgers equation generated by $X_{1}$ (scaling symmetry), $X_{2}$ (time translation), $X_{3}$ (translation of space), $X_{4}$ and $X_{5}$ (Galilei invariance). Global form of transformations are presented in Table 2. 
Table 2. Global transformations of the viscous Hopf-Burgers FDE.

\begin{tabular}{|c|c|c|c|c|}
\hline Generator & \multicolumn{4}{|c|}{ Global Transformations Associated with the Generator } \\
\hline$X_{1}^{\text {phys }}$ & $\bar{t}=t e^{2 \epsilon}$ & $\bar{x}=x e^{\epsilon}$ & $\overline{y(x) \mathrm{d} x}=y(x) \mathrm{d} x e^{\epsilon}$ & $\bar{\phi}=\phi$ \\
\hline$X_{2}^{\text {phys }}$ & $\bar{t}=t+\epsilon$, & $\bar{x}=x$ & $\overline{y(x) \mathrm{d} x}=y(x) \mathrm{d} x$ & $\bar{\phi}=\phi$ \\
\hline$X_{3}^{\text {phys }}$ & $\bar{t}=t$ & $\bar{x}=x+\epsilon$, & $\overline{y(x) \mathrm{d} x}=y(x) \mathrm{d} x$ & $\bar{\phi}=\phi$ \\
\hline$X_{4}^{\text {phys }}$ & $\bar{t}=\frac{t}{1-2 t \epsilon}$ & $\bar{x}=\frac{x}{(1-2 t \epsilon)}$ & $\overline{y(x) \mathrm{d} x}=\frac{y(x) \mathrm{d} x}{1-2 t \epsilon}$ & $\bar{\phi}=\phi \exp \left(i \frac{\epsilon}{1-2 t \epsilon} \int_{G} x y(x) \mathrm{d} x\right)$, \\
\hline$X_{5}^{\text {phys }}$ & $\bar{t}=t$ & $\bar{x}=x+2 t \epsilon$ & $\overline{y(x) \mathrm{d} x}=y(x) \mathrm{d} x$ & $\bar{\phi}=\phi \exp \left(i \epsilon \int_{G} y(x) \mathrm{d} x\right)$ \\
\hline$X_{6}^{\text {phys }}$ & $\bar{t}=t$ & $\bar{x}=x$ & $\overline{y(z) \mathrm{d} z}=y(z) \mathrm{d} z+\epsilon \mathrm{d} z$ & $\bar{\phi}=\phi$ \\
\hline$X_{7}^{\text {phys }}$ & $\bar{t}=t$ & $\bar{x}=x$ & $\overline{y(z) \mathrm{d} z}=y(z) \mathrm{d} z$ & $\bar{\phi}=\phi e^{\epsilon}+\left(1-e^{\epsilon}\right)$ \\
\hline$X_{g}^{\text {phys }}$ & $\bar{t}=t$ & $\bar{x}=x$, & $\overline{y(z) \mathrm{d} z}=y(z) \mathrm{d} z$ & $\bar{\phi}=\phi+g\left(\left[y\left(x^{\prime}\right)\right], t\right) \epsilon$. \\
\hline
\end{tabular}

\section{Conclusions}

This paper continues the work of Oberlack and Wacławczyk ( $c f . \quad[8,9])$, where the classical Lie symmetry analysis is extended from partial differential equations to equations with functional derivatives and performed in the Fourier space. Here, we introduce the procedure of applying the extended Lie symmetry analysis in the physical space. This corresponds to the case when both functional derivatives and spatial derivates with respect to the integration variable are present in the functional integro-differential equation. The method is based on the transformation of the product $y(x) \mathrm{d} x$ appearing in the integral term.

As an example, we consider the viscous Hopf-Burgers functional integro-differential equation, i.e., the functional formulation of the viscous Burgers equation. We perform the extended Lie symmetry analysis on the viscous Hopf-Burgers FDE to find the eight symmetries given in Table 1. Furthermore, we take a brief look at symmetry breaking restrictions and indicate physically-relevant symmetries, i.e., such that the transformed $\bar{\phi}$ fulfills the conditions for the characteristic functional. We see that only statistical symmetries (i.e., symmetries associated with transformations of the dependent variable $\phi$ ) are influenced if we demand $\bar{\phi}$ to be the characteristic functional. The construction of physically-relevant invariant solutions remains a task for future work. Finally, we compare the symmetries of the viscous Hopf-Burgers FDE with the symmetries of the viscous Burgers equation: we are able to rediscover all five symmetries.

The most significant result of this paper consists of demonstrating that the extended Lie symmetry analysis works for the considered functional equation and that it is not only able to rediscover symmetries of the considered equation, but also to furnish new, unknown symmetries associated with the Hopf formulation of the viscous Burgers equation having a purely statistical origin. The presented extension of the Lie symmetry analysis can be a useful tool for the analysis of FDEs with functional derivatives.

Presently, the underlying equation is the viscous Burgers equation. For future work, one might consider the Hopf-Navier-Stokes FDE and perform the extended Lie symmetry analysis on this equation to determine the moments of the solutions of the Navier-Stokes equations (see also [19]). Furthermore, one might choose a more sophisticated ansatz during the solution procedure of the determining system of equations for the infinitesimals. Hopefully, the less restrictive ansatz will lead to further new symmetries. 
We believe that the presented machinery is highly relevant to a variety of important functional differential equations and functional integro-differential equations in physics, especially in continuum mechanics. As the numerical treatment of FDEs is difficult because of the high dimensionality and since very little is known about how to treat and solve FDEs analytically, the presented methods may give a chance to treat equations that so far have been put aside because of the missing analytical methods. Additionally, unknown symmetries may be discovered, which would be pleasant, since symmetries illuminate the properties of the physical model equations.

\section{Acknowledgments}

The authors are thankful to Wolfgang Kollmann for his useful comments and discussions concerning the paper.

\section{Author Contributions}

Martin Oberlack and Marta Wacławczyk contributed analysis methods; Daniel Janocha and Marta Wacławczyk solved the determining system of equations for the infinitesimals; Daniel Janocha wrote the paper within the scope of his master's thesis.

\section{Conflicts of Interest}

The authors declare no conflict of interest.

\section{Appendix}

\section{Infinitesimals of the Dependent Variables}

For the viscous Hopf-Burgers FDE, we need the three infinitesimals $\zeta_{; t}, \zeta_{; x y(x) y(x)}$ and $\zeta_{; x x y(x)}$. $\zeta_{; t}$ is given by Equation (18):

$$
\begin{aligned}
\zeta_{; t} & =\frac{\partial \eta_{\phi}}{\partial t}+\phi_{, t}\left(\frac{\partial \eta_{\phi}}{\partial \phi}-\frac{\partial \xi_{t}}{\partial t}\right)-\left(\phi_{, t}\right)^{2} \frac{\partial \xi_{t}}{\partial \phi}-\int_{G} \phi_{, y\left(x^{\prime}\right)} \frac{\partial \xi_{y\left(x^{\prime}\right)} \mathrm{d} x^{\prime}}{\partial t}-\int_{G} \phi_{, y\left(x^{\prime}\right)} \phi_{, t} \frac{\partial \xi_{y\left(x^{\prime}\right)} \mathrm{d} x^{\prime}}{\partial \phi} \\
& -\int_{G} \phi_{, x^{\prime} y\left(x^{\prime}\right)} \frac{\partial \xi_{x^{\prime}}}{\partial t} y\left(x^{\prime}\right) \mathrm{d} x^{\prime}-\int_{G} \phi_{, x^{\prime} y\left(x^{\prime}\right)} \phi_{, t} \frac{\partial \xi_{x^{\prime}}}{\partial \phi} y\left(x^{\prime}\right) \mathrm{d} x^{\prime} .
\end{aligned}
$$

$\zeta_{; x y(x) y(x)}$ and $\zeta_{; x x y(x)}$ are given below:

$$
\begin{aligned}
\zeta_{; x y(x) y(x)=} & \frac{\partial^{3} \eta_{\phi}}{\partial x \partial(y(x) \mathrm{d} x)^{2}}+2 \phi_{, y(x)} \frac{\partial^{3} \eta_{\phi}}{\partial x \partial y(x) \mathrm{d} x \partial \phi}-\phi_{, t} \frac{\partial^{3} \xi_{t}}{\partial x \partial(y(x) \mathrm{d} x)^{2}} \\
& -2 \phi_{, t} \phi_{, y(x)} \frac{\partial^{3} \xi_{t}}{\partial x \partial y(x) \mathrm{d} x \partial \phi}-\int_{G} \phi_{, y(z)} \frac{\partial^{3} \xi_{y(z)} \mathrm{d} z}{\partial x \partial(y(x) \mathrm{d} x)^{2}} \\
& -2 \int_{G} \phi_{, y(z)} \phi_{, y(x)} \frac{\partial^{3} \xi_{y(z)} \mathrm{d} z}{\partial x \partial y(x) \mathrm{d} x \partial \phi}+\left(\phi_{, y(x)}\right)^{2} \frac{\partial^{3} \eta_{\phi}}{\partial x \partial \phi^{2}}-\phi_{, t}\left(\phi_{, y(x)}\right)^{2} \frac{\partial^{3} \xi_{t}}{\partial x \partial \phi^{2}} \\
& -\int_{G} \phi_{, y(z)}\left(\phi_{, y(x)}\right)^{2} \frac{\partial^{3} \xi_{y(z)} \mathrm{d} z}{\partial x \partial \phi^{2}}-2 \phi_{, t y(x)} \frac{\partial^{2} \xi_{t}}{\partial x \partial y(x) \mathrm{d} x}-2 \phi_{, y(x)} \phi_{, t y(x)} \frac{\partial^{2} \xi_{t}}{\partial x \partial \phi}
\end{aligned}
$$


$-2 \int_{G} \phi_{, y(z) y(x)} \frac{\partial^{2} \xi_{y(z)} \mathrm{d} z}{\partial x \partial y(x) \mathrm{d} x}-2 \int_{G} \phi_{, y(x)} \phi_{, y(z) y(x)} \frac{\partial^{2} \xi_{y(z)} \mathrm{d} z}{\partial x \partial \phi}-\phi_{, x y(x)} \frac{\partial^{2} \xi_{x}}{\partial x \partial y(x) \mathrm{d} x}$

$-\phi_{, y(x)} \phi_{, x y(x)} \frac{\partial^{2} \xi_{x}}{\partial x \partial \phi}+\phi_{, y(x) y(x)} \frac{\partial^{2} \eta_{\phi}}{\partial x \partial \phi}-\phi_{, t} \phi_{, y(x) y(x)} \frac{\partial^{2} \xi_{t}}{\partial x \partial \phi}$

$-\int_{G} \phi_{, y(z)} \phi_{, y(x) y(x)} \frac{\partial^{2} \xi_{y(z)} \mathrm{d} z}{\partial x \partial \phi}$

$+2 \phi_{, x y(x)} \frac{\partial^{2} \eta_{\phi}}{\partial y(x) \mathrm{d} x \partial \phi}-2 \phi_{, t} \phi_{, x y(x)} \frac{\partial^{2} \xi_{t}}{\partial y(x) \mathrm{d} x \partial \phi}-\int_{G} \phi_{, x y(z)} \frac{\partial^{2} \xi_{y(z)} \mathrm{d} z}{\partial(y(x) \mathrm{d} x)^{2}}$

$-2 \int_{G} \phi_{, x y(z)} \phi_{, y(x)} \frac{\partial^{2} \xi_{y(z)} \mathrm{d} z}{\partial y(x) \mathrm{d} x \partial \phi}-2 \int_{G} \phi_{, y(z)} \phi_{, x y(x)} \frac{\partial^{2} \xi_{y(z)} \mathrm{d} z}{\partial y(x) \mathrm{d} x \partial \phi}$

$+2 \phi_{, y(x)} \phi_{, x y(x)} \frac{\partial^{2} \eta_{\phi}}{\partial \phi^{2}}-2 \phi_{, t} \phi_{, y(x)} \phi_{, x y(x)} \frac{\partial^{2} \xi_{t}}{\partial \phi^{2}}-\int_{G} \phi_{, x y(z)}\left(\phi_{, y(x)}\right)^{2} \frac{\partial^{2} \xi_{y(z)} \mathrm{d} z}{\partial \phi^{2}}$

$-2 \int_{G} \phi_{, y(z)} \phi_{, y(x)} \phi_{, x y(x)} \frac{\partial^{2} \xi_{y(z)} \mathrm{d} z}{\partial \phi^{2}}-2 \phi_{, x y(x)} \phi_{, t y(x)} \frac{\partial \xi_{t}}{\partial \phi}$

$-2 \int_{G} \phi_{, x y(x)} \phi_{, y(z) y(x)} \frac{\partial \xi_{y(z)} \mathrm{d} z}{\partial \phi}-\left(\phi_{, x y(x)}\right)^{2} \frac{\partial \xi_{x}}{\partial \phi}-\int_{G} \phi_{, x y(z)} \phi_{, y(x) y(x)} \frac{\partial \xi_{y(z)} \mathrm{d} z}{\partial \phi}$

$-2 \phi_{, x t y(x)} \frac{\partial \xi_{t}}{\partial y(x) \mathrm{d} x}-2 \phi_{, y(x)} \phi_{, x t y(x)} \frac{\partial \xi_{t}}{\partial \phi}-\phi_{, x x y(x)} \frac{\partial \xi_{x}}{\partial y(x) \mathrm{d} x}-\phi_{, y(x)} \phi_{, x x y(x)} \frac{\partial \xi_{x}}{\partial \phi}$

$-2 \int_{G} \phi_{, x y(z) y(x)} \frac{\partial \xi_{y(z)} \mathrm{d} z}{\partial y(x) \mathrm{d} x}-2 \int_{G} \phi_{, y(x)} \phi_{, x y(z) y(x)} \frac{\partial \xi_{y(z)} \mathrm{d} z}{\partial \phi}+\phi_{, x y(x) y(x)} \frac{\partial \eta_{\phi}}{\partial \phi}$

$-\phi_{, t} \phi_{, x y(x) y(x)} \frac{\partial \xi_{t}}{\partial \phi}-\int_{G} \phi_{, y(z)} \phi_{, x y(x) y(x)} \frac{\partial \xi_{y(z)} \mathrm{d} z}{\partial \phi}-\phi_{, t y(x) y(x)} \frac{\partial \xi_{t}}{\partial x}$

$-\int_{G} \phi_{, y(z) y(x) y(x)} \frac{\partial \xi_{y(z)} \mathrm{d} z}{\partial x}-\phi_{, x y(x) y(x)} \frac{\partial \xi_{x}}{\partial x}-\phi_{, x x y(x)} \frac{\partial \xi_{x}}{\partial y(x) \mathrm{d} x}$

$-\phi_{, x y(x)} \frac{\partial^{2} \xi_{x}}{\partial x \partial y(x) \mathrm{d} x}-\phi_{, x x y(x)} \phi_{, y(x)} \frac{\partial \xi_{x}}{\partial \phi}-\phi_{, x y(x)} \phi_{, x y(x)} \frac{\partial \xi_{x}}{\partial \phi}-\phi_{, x y(x)} \phi_{, y(x)} \frac{\partial^{2} \xi_{x}}{\partial \phi \partial x}$

$-\int_{G} \phi_{, y(x)} \phi_{, z y(z)} y(z) \frac{\partial^{3} \xi_{z}}{\partial x \partial \phi \partial y(x) \mathrm{d} x} \mathrm{~d} z-2 \int_{G} \phi_{, z y(x) y(z)} y(z) \frac{\partial^{2} \xi_{z}}{\partial x \partial \partial y(x) \mathrm{d} x} \mathrm{~d} z$

$-\int_{G} \phi_{, z x y(z)} y(z) \frac{\partial^{2} \xi_{z}}{(\partial y(x) \mathrm{d} x)^{2}} \mathrm{~d} z-\int_{G} \phi_{, z y(z)} y(z) \frac{\partial^{3} \xi_{z}}{\partial x(\partial y(x) \mathrm{d} x)^{2}} \mathrm{~d} z$

$-\int_{G} \phi_{, x y(x)} \phi_{, z y(z)} y(z) \frac{\partial^{2} \xi_{z}}{\partial \phi \partial y(x) \mathrm{d} x} \mathrm{~d} z-\int_{G} \phi_{, y(x)} \phi_{, z x y(z)} y(z) \frac{\partial^{2} \xi_{z}}{\partial \phi \partial y(x) \mathrm{d} x} \mathrm{~d} z$

$-2 \int_{G} \phi_{, z x y(x) y(z)} y(z) \frac{\partial \xi_{z}}{\partial y(x) \mathrm{d} x} \mathrm{~d} z-2 \int_{G} \phi_{, z x y(x) y(z)} \phi_{, y(x)} y(z) \frac{\partial \xi_{z}}{\partial \phi} \mathrm{d} z$

$-2 \int_{G} \phi_{, z y(x) y(z)} \phi_{, x y(x)} y(z) \frac{\partial \xi_{z}}{\partial \phi} \mathrm{d} z-\int_{G} \phi_{, x y(x) y(x)} \phi_{, z y(z)} y(z) \frac{\partial \xi_{z}}{\partial \phi} \mathrm{d} z$ 


$$
\begin{aligned}
\zeta_{; x x y(x)}= & \frac{\partial^{3} \eta_{\phi}}{\partial x^{2} \partial y(x) \mathrm{d} x}-\phi_{, t} \frac{\partial^{3} \xi_{t}}{\partial x^{2} \partial y(x) \mathrm{d} x}-\int_{G} \phi_{, y(z)} \frac{\partial^{3} \xi_{y(z)} \mathrm{d} z}{\partial x^{2} \partial y(x) \mathrm{d} x}-\int_{G} \phi_{, y(z) y(x)} \frac{\partial^{3} \xi_{y(z)} \mathrm{d} z}{\partial x^{2} \partial \phi} \\
& +\phi_{, x y(x)} \frac{\partial^{2} \eta_{\phi}}{\partial x \partial \phi}-2 \phi_{, t} \phi_{, x y(x)} \frac{\partial^{2} \xi_{t}}{\partial x \partial \phi}-2 \int_{G} \phi_{, x y(z)} \frac{\partial^{2} \xi_{y(z)} \mathrm{d} z}{\partial x \partial y(x) \mathrm{d} x}-\int_{G} \phi_{, x y(z)} \phi_{, y(x)} \frac{\partial^{2} \xi_{y(z)} \mathrm{d} z}{\partial x \partial \phi} \\
& -2 \int_{G} \phi_{, y(z)} \phi_{, x y(x)} \frac{\partial^{2} \xi_{y(z)} \mathrm{d} z}{\partial x \partial \phi}-\int_{G} \phi_{, y(z) y(x)} \frac{\partial^{2} \xi_{y(z)} \mathrm{d} z}{\partial x^{2}}-\phi_{, x y(x)} \frac{\partial^{2} \xi_{x}}{\partial x^{2}} \\
& -\int_{G} \phi_{, x y(z)} \phi_{, y(x)} \frac{\partial^{2} \xi_{y(z)} \mathrm{d} z}{\partial x \partial \phi}-2 \int_{G} \phi_{, x y(z)} \phi_{, x y(x)} \frac{\partial \xi_{y(z)} \mathrm{d} z}{\partial \phi}+\phi_{, x x y(x)} \frac{\partial \eta_{\phi}}{\partial \phi}-\phi_{, t} \phi_{, x x y(x)} \frac{\partial \xi_{t}}{\partial \phi} \\
& -\int_{G} \phi_{, x x y(z)} \frac{\partial \xi_{y(z)} \mathrm{d} z}{\partial y(x) \mathrm{d} x}-\int_{G} \phi_{, x x y(z)} \phi_{, y(x)} \frac{\partial \xi_{y(z)} \mathrm{d} z}{\partial \phi}-\int_{G} \phi_{, y(z)} \phi_{, x x y(x)} \frac{\partial \xi_{y(z)} \mathrm{d} z}{\partial \phi} \\
& -2 \phi_{, x x y(x)} \frac{\partial \xi_{x}}{\partial x}-\int_{G} \phi_{, x y(z) y(x)} \frac{\partial \xi_{y(z)} \mathrm{d} z}{\partial x} \\
& -\phi_{, t x y(x)} \frac{\partial \xi_{t}}{\partial x}-\int_{G} \phi_{, y(z) x y(x)} \frac{\partial \xi_{y(z)} \mathrm{d} z}{\partial x}-\phi_{, t} \phi_{, y(x)} \frac{\partial^{3} \xi_{t}}{\partial x^{2} \partial \phi}-\phi_{, t y(x)} \frac{\partial^{2} \xi_{t}}{\partial x^{2}}-\phi_{, x t y(x)} \frac{\partial \xi_{t}}{\partial x} \\
& -\int_{G} \phi_{, z y(z)} y(z) \frac{\partial^{3} \xi_{z}}{\partial x^{2} \delta y(x)} \mathrm{d} z-\int_{G} \phi_{, x x z y(z)} y(z) \frac{\delta \xi_{z}}{\delta y(x)} \mathrm{d} z-\int_{G} \phi_{, z y(z)} \phi_{, x x y(x)} y(z) \frac{\partial \xi_{z}}{\partial \phi} \mathrm{d} z \\
& -2 \int_{G} \phi_{, x z y(z)} y(z) \frac{\partial^{2} \xi_{z}}{\partial x \delta y(x)} \mathrm{d} z .
\end{aligned}
$$

\section{References}

1. Oberlack, M.; Rosteck, A. New statistical symmetries of the multi-point equations and its importance for turbulent scaling laws. Disc. Cont. Dyn. Sys. Ser. S 2010, 3, 451-471.

2. Lundgren, T.S. Distribution functions in the statistical theory of turbulence. Phys. Fluids 1967, 10, 969-975.

3. Wacławczyk, M.; Staffolani, N.; Oberlack, M.; Rosteck, A.; Wilczek, M.; Friedrich, R. Statistical Symmetries of the Lundgren-Monin-Novikov Hierarchy. Phys. Rev. E 2014, 90, doi:10.1103/PhysRevE.90.013022.

4. Hopf, E. Statistical Hydromechanics and Functional Calculus. J. Ration. Mech. Anal. 1952, 1, 87-123.

5. Hosokawa, I. Monin-Lundgren hierarchy versus the Hopf equation in the statistical theory of turbulence. Phys. Rev. E 2006, 73, doi:10.1103/PhysRevE.73.067301.

6. Alt, H.W. Lineare Funktionalanalysis; Springer-Verlag: Berlin, Germany, 2012.

7. Gelfand, I.M.; Fomin, S.W. Calculus of Variations; Prentice Hall: Upper Saddle River, NJ, USA, 1963.

8. Oberlack, M.; Wacławczyk, M. On the extension of Lie group analysis to functional differential equations. Arch. Mech. 2006, 58, 597-618.

9. Wacławczyk, M.; Oberlack, M. Application of the extended Lie group analysis to the Hopf functional formulation of the Burgers equation. J. Math. Phys. 2013, 54, doi:10.1063/1.4812803.

10. Hosokawa, I.; Yamamoto, K. Numerical Study of the Burgers' Model of Turbulence Based on the Characteristic Functional Formalism. Phys. Fluids 1970, 13, 1683-1692. 
11. Hosokawa, I.; Yamamoto, K. Energy decay of Burgers' model of turbulence. Phys. Fluids 1976, $19,1423-1424$.

12. Grebenev, N.N.; Nazarenko, S.V.; Medvedev S.B.; Schwab I.V.; Chirkunov, Y.A. Self-similar solution in the Leith model of turbulence: anomalous power law and asymptotic analysis. J. Phys. A Math. Theor. 2014, 47, doi:10.1088/1751-8113/47/2/025501.

13. Ibragimov, N.H. CRC Handbook of Lie Group Analysis of Differential Equations, Volume 1: Symmetries, Exact Solutions and Conservation Laws; CRC Press: Boca Raton, FL, USA, 1994.

14. Klauder, J.R. A Modern Approach to Functional Integration; Birkhäuser: Basel, Switzerland, 2011.

15. Ibragimov, N.H.; Kovalev, V.F.; Pustovalov V.V. Symmetries of integro-differential equations: A survey of methods illustrated by the Benny equations. Nonlinear Dyn. 2002, 28, 135-153.

16. Ibragimov, N.H. CRC Handbook of Lie Group Analysis of Differential Equations, Volume 3: New Trends in Theoretical Developments and Computational Methods; CRC Press: Boca Raton, FL, USA, 1996.

17. Zawistowski, Z.J. Symmetries of Integro-Differential Equations. Rep. Math. Phys. 2001, 48, 269-275.

18. Özer, T. Symmetry group analysis of Benney system and an application for shallow-water equations. Mech. Res. Commun. 2005 , 32, 241-254.

19. Oberlack, M.; Wacławczyk, M.; Rosteck, A.; Avsarkisov, V. Symmetries and their importance for statistical turbulence theory. Mech. Eng. Rev. 2015, 2, doi:10.1299/mer.15-00157.

(c) 2015 by the authors; licensee MDPI, Basel, Switzerland. This article is an open access article distributed under the terms and conditions of the Creative Commons Attribution license (http://creativecommons.org/licenses/by/4.0/). 